Running Head: PARENTING AND ADOLESCENT SELF-CONTROL

Parenting and Adolescent Self-Control: A Three-Level Meta-Analysis

Jian-Bin $\mathrm{Li}^{*}$

Department of Early Childhood Education, The Education University of Hong Kong, Hong Kong Centre for Child and Family Science, The Education University of Hong Kong, Hong Kong E-mail: lijianbin@eduhk.hk

Yayouk E Willems*

Department of Biological Psychology, Vrije Universiteit Amsterdam, Amsterdam

Amsterdam Public Health Institute, Amsterdam, the Netherlands

Department of Interdisciplinary Social Sciences, Utrecht University, Utrecht

E-mail: y.e.willems@,vu.nl

F. Marijn Stok

Department of Interdisciplinary Social Sciences, Utrecht University, Utrecht

E-mail: f.m.stok@uu.nl

Maja Deković

Department of Clinical Child and Family Studies, Utrecht University, Utrecht

E-mail: M.Dekovic@uu.nl

Meike Bartels

Department of Biological Psychology, Vrije Universiteit Amsterdam, Amsterdam

Amsterdam Public Health Institute, Amsterdam, the Netherlands

Neuroscience Amsterdam, Amsterdam, the Netherlands

E-mail: m.bartels@,vu.nl

Catrin Finkenauer

Department of Interdisciplinary Social Sciences, Utrecht University, Utrecht

E-mail: c.finkenauer@uu.nl

*Author note: JBL and YEW contributed equally to the paper and are co-first authors.

Corresponding author: 
Jian-Bin Li, Ph.D.

Assistant Professor

Department of Early Childhood Education

The Education University of Hong Kong

10, Lo Ping Road,

Tai Po, New Territories, Hong Kong

E-mail: lijianbin@eduhk.hk

Tel: +85229487587

Fax: +85229487160

Yayouk E. Willems

Department of Biological Psychology

Vrije Universiteit

van der Boechorststraat 1, 1081 BT, Amsterdam, The Netherlands.

E-mail: y.e.willems@vu.nl,

Tel: +31205984382

Acknowledgement of financial support:

J.B. Li is supported by FEHD's Internationalization \& Exchange Research Scheme of the Education University of Hong Kong. Y. Willems is supported by NWO (Research Talent, 406-15-132), and by the Amsterdam Public Health Personalized Medicine Travel Grant. M. Bartels is financially supported by a VU University Research Chair position; the "Aggression in Children: Unraveling gene-environment interplay to inform Treatment and InterventiON strategies" project (ACTION). ACTION receives funding from the European Union Seventh Framework Program (FP7/2007-2013) under grant agreement no 602768; The MARIE Skłodowska-CURIE ACTIONS Innovative Training Network (ITN) "CAPICE" (Childhood and Adolescence Psychopathology: unravelling the complex etiology by a large Interdisciplinary Collaboration in Europe) (H2020-MSCA-ITN-2016 721567-CAPICE). 


\begin{abstract}
Self-control is a crucial psychological function and plays a significant role in positive youth development. While numerous self-control challenges occurs during adolescence, some adolescents better control themselves than others. Parenting is considered a critical factor distinguishing adolescents with good and poor self-control, but existing findings are inconsistent. The meta-analysis summarized the overall relationship between parenting and self-control among adolescents aged 10 to 22 . One hundred and ninety-one articles reporting 1,540 effect sizes $(N=164,459)$ were included in the analysis. The results showed that parenting is associated with adolescents' self-control both concurrently $(r=.204, p<.001)$ and longitudinally $(r=.157, p<.001)$. Longitudinal studies also revealed that adolescents' self-control influences subsequent parenting $(r=.155, p<.001)$. Moderator analyses showed that the effect sizes were largely invariant across cultures, ethnicities, age of adolescents, and parent and youth gender. Our results point to the importance of parenting in individual differences in adolescent self-control and vice versa.
\end{abstract}

Key words: parenting; parent-child relationship; self-control; adolescence. 


\section{Parenting and Adolescent Self-Control: A Three-Level Meta-Analysis}

Adolescence is a distinct and pivotal period of life with significant changes and growth at every level of functioning. What happens during adolescence has profound and long-lasting implications for youth's trajectories of economic security, health, and well-being and development into later life (Patton et al., 2016). It is also a sensitive period featured by increasing prevalence of risk behaviors jeopardizing youth's physical and psychological functioning across their life course (Arnett, 1992, 1999; Casey, Jones, \& Hare, 2008; Steinberg, 2004). Consequently, numerous studies investigated what factors counteract adolescents' problem behaviors and self-control is found to be such a factor (Moffit et al., 2011; Caspi et al., 2017). Self-control - defined as one's ability to alter dominant responses to abide by social values, moral norms, and to support the pursuit of long-term goals (Baumeister, Vohs, \& Tice, 2007; Tangney, Baumeister, \& Boone, 2004) - plays a key role in positive youth development and is widely studied across disciplines such as economics, public health and neuroscience (Caspi et al., 2017; Figner et al., 2010; Hare, Camerer, \& Rangel, 2009). It helps adolescents through a stage marked by a range of normative biological and social challenges (Crone \& Dahl, 2012), increases in risk-taking and social reward seeking behavior (Boyer, 2006; Smetana, Campione-Barr, \& Metzger, 2006), and heightened emotional turmoil (Steinberg \& Morris, 2001).

To illustrate, adolescents with higher levels of self-control use less drugs and alcohol (Vazsonyi, Trejos-Castillo, \& Huang, 2006), experience fewer emotional and behavioral problems (Finkenauer, Engels, \& Baumeister, 2005; Li, Delvecchio, Lis, Nie, \& Di Riso, 2015, Situ, Li, \& Dou, 2016; Vazsonyi \& Belliston, 2007), show more prosocial behavior 
(Nie, Li, \& Vazsonyi, 2016; Padilla-Walker, \& Christensen, 2011), and are more likely to achieve better academic accomplishments (Duckworth \& Seligman, 2005; Galla \& Duckworth, 2015) than adolescents with lower levels of self-control. Whereas self-control generally continues to improve throughout adolescence in terms of neural and psychological development (Casey, Jones, \& Hare, 2008; Giedd, 2004; Romer, Duckworth, Sznitman, \& Park, 2010), some adolescents show better self-control than others. To enhance our understanding of how these individual differences in self-control come about during the unique period of adolescence, the present meta-analysis sought to examine the relation between parenting and self-control across adolescence by considering the joint contribution of adolescents and parents to adolescent development.

Theorists agree that parenting is an important factor associated with individual differences in self-control (Bridgett, Burt, Edwards, \& Deater-Deckard, 2015; Gottfredson \& Hirschi, 1990; Kochanska, 1993; Kopp, 1982; Sameroff, 2010). More specifically, prior studies found that positive parenting (e.g., monitoring, consistent discipline, parental warmth and support, positive control, authoritative parenting) and a strong parent-child relationship (e.g., secure attachment, close parent-child bonding, high quality of relationship) are related to better self-control. Conversely, negative parenting (e.g., inconsistent discipline, harsh parenting, coercive parenting, physical punishment, negative control, authoritarian parenting) and a weak parent-child relationship are associated with lower self-control in early and middle childhood (see meta-analyses: Davis, Bilms, \& Suveg, 2017; Karreman, van Tuijl, van Aken, \& Deković, 2006; Pallini et al., 2018). However, studies extending this work to adolescence yield mixed findings. While some studies report robust cross-sectional and 
longitudinal associations between parenting and self-control throughout adolescence (Hay, 2001; Hope et al., 2003; Özdemir et al., 2013; Vazsonyi \& Belliston, 2007), others report only significant concurrent associations (Baardstu et al., 2017; Craig, 2015; Moilanen, Rasmussen, Padilla-Walker, 2014; Pallini et al., 2018), and some studies find significant associations for some parenting dimensions but not for others (Finkenauer et al., 2005; Vazsonyi et al., 2016). As such, previous findings regarding the magnitude and the direction of the association between parenting and self-control in adolescence are not conclusive.

These inconsistent findings might be explained by the notion that parenting is most strongly associated with self-control during early and middle childhood than in other developmental period (Kochanska, 1993; Gottfredson \& Hirschi, 1990; Hay \& Meldrum, 2016; Kopp, 1982; Meldrum, Young, Hay, \& Flexon, 2012; Vazsonyi \& Jiskrova, 2017). During early and middle childhood, children rely mostly on their parents for decision making, behavior guidance, and emotion regulation, and parents assist children with their self-regulatory capacities because they provide an environment and experience assisting children in regulating their inner feelings and behaviors (Kopp, 1982; Sameroff, 2010). When entering teenage years, adolescents transit into a phase with relative independence as they gradually desire more autonomy from parents: adolescents interact less with parents and more with peers, and are more likely to negotiate with parents about social customs and/or conventions instead of merely following parents' guidance (Steinberg \& Silk, 2002). As such, in the teenage years the influence of parenting on the development of self-control may decrease (Farley \& Kim-Spoon, 2014; Hay \& Meldrum, 2016; Tiberio et al., 2016). However, while adolescents may feel they are independent and responsible 
individuals, parents generally remain important figures adolescents turn to for emotional and financial support when needed (Buist, Deković, Meeus, \& van Aken, 2002; Li, Delvecchio, Miconi, Salcuni, \& Di Riso, 2014). These social changes and developmental transitions in adolescence yield an important question: Is parenting still important for self-control during adolescence? Asking this question for adolescence is all the more important as scientists increasingly recognize that development and growth processes that have their beginning in early adolescence continue into the twenties (Sawyer, Azzopardi, Wickremarathne, \& Patton, 2018). Additionally, societal changes lead to delays in adopting adult roles (e.g., life expectancy, longer educational trajectories), research call for extending our conceptualization of adolescence as ranging from early adolescence to the early twenties (Arroyo, Payne, Brown, \& Manning,. 2013; Sawyer et al., 2018). In light of these considerations, researchers have pointed out that the adolescence period covers "a greater proportion of the life course with greater relevance for human development than ever before" (Patton et al., 2018, p. 458). Thus, it would be crucial to take stock of the empirical findings and inform the field about the association between parenting and self-control across the course of adolescence.

The current meta-analysis adds to previous meta-analyses in several important ways. Previous meta-analyses regarding the association between parenting and self-control focused either on young children (Davis et al., 2017; Karreman et al., 2006) or on specific parenting practice or indicators (i.e., parent-child synchrony, Davis et al., 2017; attachment security status, Pallini et al., 2018). Moreover, crucially, existing meta-analyses failed to take possible reciprocal effects of self-control on subsequent parenting into account (Sameroff, 2010). Especially during adolescence, it is important to consider evocative effects of adolescent 
self-control on parenting, which may play a particularly important role in promoting and maintaining its continuity across adolescence. Indeed, it may well be that early adolescent self-control evokes responses from the parental environment that reinforce the child's tendencies over time (e.g., Caspi \& Roberts, 2001). Finally, existing studies did not apply three-level analyses, a novel technique to better distinguish variance at the sample, within-study, and between-study level to provide a more accurate estimate of the results (Assink \& Wibbelink, 2016), such as taking into account dependency between different effect sizes extracted from the same study without losing the rich information of a study that contains multiple effect sizes (Cheung, 2014; Van den Noortgate et al., 2013).

To our knowledge, a comprehensive review synthesizing empirical studies on the association between broad types of parenting and self-control among adolescents is still lacking. This is crucial because the interplay between individual and social environment is at the core of the development of self-control, especially across the adolescent period. Adolescents increasingly become active agents of their own development, and their levels of self-control are likely to evoke responses from their parents generating reciprocal influence of persons and environments (Eisenberg et al., 2005; Sameroff, 2010; Tiberio et al., 2016). In order to clarify whether parenting is associated with self-control across adolescence and identify factors that influence this association, this pre-registered meta-analysis ${ }^{1}$ aimed to answer the following questions: (1) Is parenting important to self-control of adolescents aged 10 to 22 years? (2) Does adolescents' self-control influence subsequent parenting? Additionally, and third, we explored whether theoretical and methodological factors included

\footnotetext{
${ }^{1}$ we pre-registered this research on https://aspredicted.org/gs2zi.pdf
} 
in previous meta-analyses about self-control moderate the magnitude of the association between parenting and self-control in adolescence (e.g., Hoeve et al., 2009; Karreman et al., 2006; Vazsonyi et al., 2017). Investigating these questions allows us to aggregate diverse individual study results to identify the overall mean effect and examine the role of possible moderators on the magnitude of this effect. Doing so generates insights about self-control development over the course of adolescence and elucidates gaps that should be given attention in future research aiming to understand individual differences in this important capacity.

\section{Conceptualization of self-control}

Research on self-control spans the social and behavioral sciences (Duckworth \& Steinberg, 2015). The nomenclature of self-control varies by theoretical tradition, with social psychologists and criminologists referring more often to self-control (e.g., Gottfredson \& Hirschi, 1990; Tangney, Baumeister \& Boone, 2004), while developmental psychologists often refer to concepts such as self-regulation and effortful control (e.g., Bridgett, Burt, Edwards, \& Deater-Deckard, 2015; Eisenberg et al., 2003, 2005; Kopp, 1982).

Although there is considerable dissent in the literature about how to label or define self-control, existing theories and findings generally agree that self-control, self-regulation, and effortful control tap into the same capacity. The common thread going through these concepts is the involvement of voluntary self-governance, an ability one consciously uses to manage one's cognition, emotion, and behavior (Bridgett et al., 2015; Duckworth \& Kern, 2011; Nigg, 2017). Evidence from various aspects supports this view. First, studies applying factor structure analyses combining tasks attributed to different conceptualizations of 
self-control are best presented by a single factor model (Allan, Hume, Allan, Farrington, \& Lonigan, 2014; Allan \& Lonigan, 2011). Second, a meta-analysis which summarized the convergence of a number of self-control measures derived from different theories, perspectives, and approaches revealed that these measures are moderately convergent (Duckworth \& Kern, 2011). Additionally, neuroscientific research showed overlapping neural substrates associated with these concepts (Fan, Flombaum, McCandliss, Thomas, \& Posner, 2003; Garavan, Ross, Murphy, Roche, \& Stein, 2002). Therefore, in this study, we included self-control as well as these analogous terms, referring to them overall as 'self-control', as done by prior meta-analyses on self-control (Davis et al., 2017; De Ridder et al., 2012; Karreman et al., 2006; Pallini et al., 2018; Vazsonyi et al., 2017).

\section{The association between parenting and self-control}

Parenting is a multifaceted construct containing various terms reflecting different aspects of parenting processes. In this research, we mainly focused on three broad dimensions of parenting (i.e., positive parenting practices, negative parenting practices, and parent-child relationship) in relation to adolescent self-control. Positive parenting refers to parental behaviors that reflect positive control and warmth, such as parental warmth, monitoring, supervision, consistent discipline, parental support, authoritative parenting (Darling \& Steinberg, 1993; O’Connor, 2002). Negative parenting refers to behaviors that reflect negative control and hostility, such as harsh parenting, inconsistent discipline, coercive punishment, authoritarian parenting, and permissive parenting (Darling \& Steinberg, 1993; O'Connor, 2002). Parent-child relationship refers to children's emotional bond with parents (Cassidy, 1994); this construct is also often labeled parent-child attachment (Bowlby, 1969), 
or parent-child bonding (Gottfredson \& Hirschi, 1990) across the literature.

The reasons for us to concentrate on these three broad types of parenting are threefold. First, the parenting literature has primarily focused on two broad categories of parenting (parenting behavior/practices, Darling \& Steinberg, 1993) and an emotional relationship/bond between parent and child (Belsky, 1984; Bowlby, 1969), with the former further being divided into positive and negative parenting practices (O'Connor, 2002). Second, these three parenting types correspond to existing theories from various disciplines. For example, the general theory of crime postulates that self-control is nurtured by positive parenting practices (monitoring, consistent discipline) and a close parent-child relationship, while negative parenting practices (excessive punishment, permissive parenting) and weak parent-child relationship undermine self-control development (Gottfredson \& Hirschi, 1990). The process model (Belsky, 1984) also suggests that parenting and parent-child relationship are different constructs within the family system that affect child development (Belsky, 1984). Third, as shown in Table 1 of supplemental materials, we have identified a host of specific parenting terms, with some (e.g., conflict, monitoring, authoritative) much more frequently used to examine the "parenting - self-control" association than others (e.g., neglect, overprotection, alienation). An advantage of focusing on the three broad types of parenting dimensions is that it allows us to group many relevant studies together, ensuring sufficient statistical power. If we focused on each specific term separately, it would not be possible to conduct moderation analyses because many terms would only appear in the literature a few times (e.g., neglect, overprotection, alienation) and statistical power would be too low. Hence, in this study we focused on the three types of parenting dimension, a strategy adopted by prior meta-analyses 
on parenting and child outcomes (e.g., Davis et al., 2017; Karreman et al., 2006; Pallini et al., 2018; Slagt, Dubas, Deković, \& van Aken, 2016).

\section{The influence of parenting on self-control}

Parents who employ positive parenting strategies provide clear standards for behavior (Sroufe, 1996), monitor and discipline their children's undesirable behavior timely and consistently (Gottfredson \& Hirschi, 1990), and guide them to work through problems themselves (Putnam, Spritz, \& Stifter, 2002; Strand, 2002), all of which help them gradually internalize others' rules and expectation of what are appropriate behaviors and may foster the development of self-control of adolescents. Many studies linked positive parenting with youth's self-control, generally finding that positive parenting relates to good self-control development in adolescents (e.g., Finkenauer et al., 2005; Hay, 2001; Hope et al., 2003; Özdemir et al., 2013; Vazsonyi \& Belliston, 2007). However, it warrants attention that some inconsistent findings emerge in terms of significance and magnitude. For example, in their study Baardstu et al., (2017) found no significant longitudinal associations between positive parenting and self-control over the course of early adolescence.

Parents who use negative parenting strategies are likely to deprive youths of opportunities to figure out self-control strategies independently (Grolnick, McMenamy, \& Kurowski, 1999), provide youths with little or no guidance to self-regulate when needed (Baumrind, 1991), monitor and discipline youths' undesirable behavior inconsistently or ineffectively (Gottfredson \& Hirschi, 1990), and create a stressful family environment that jeopardizes children's internalization of social rules (Kochanska \& Aksan, 1995; Silverman \& Ragusa, 1990). Negative parenting therefore provides a context that hampers the 
development of self-control in adolescence. Consistent with this suggestion, prior research has generally found that negative parenting is related to low self-control (e.g., Brody \& Ge, 2001; Cheung \& Cheung, 2010; Feldman \& Wentzel, 1990; Hallquist, Hipwell, \& Stepp, 2015; Simons, Simons, Chen, Brody, \& Lin, 2007). Again, however, the evidence is inconsistent. For instance, prior research found that mothers' authoritarian and permissive parenting style and fathers' authoritarian and permissive parenting style were not significantly related to their adolescent children's self-control (Jabagchourian, Sorkhabi, Quach, \& Strage, 2014).

For parent-child relationship, parents who successfully establish close bonds foster children to develop better emotional regulation ability, which lays the foundation for further development of self-control (Bowlby, 1969; Cassidy, 1994; Kopp, 1982; Pallini et al., 2018). A close parent-child relationships (in some studies defined as secure parent-child attachment $)^{2}$ during adolescence serve as the foundation for parents to monitor, recognize and discipline adolescents' behavior (Gottfredson \& Hirschi, 1990) and, in turn, encourages children to share information about their daily activities with their parents (Kerr \& Stattin, 2000; Parke, 2004). A close parent-child relationship thereby provides a context that is conducive to parental scaffolding and teaching of self-control. Consistent with this suggestion, numerous studies found that secure attachment is associated with better self-control (e.g., Alvarez-Rivera \& Fox, 2010; Li et al., 2015; Nie, Li, \& Vazsonyi, 2016; Wills, Gibbons, Gerrard, Murry, \& Brody, 2003; You \& Kim, 2016). Nevertheless, the strength of the

\footnotetext{
2 Attachment during adolescence is usually understood/measured as close parent-child relationship and can be considered , as the continuum of childhood attachment rather than the attachment dimensions as by Bowlby \& Ainsworth (Armsden \& Greenberg, 1987). In this study we therefore used the term "parent-child relationship" instead of "parent-child attachment", but in literature search and coding we categorized relationship and attachment as the same subtype, namely 'parent-child relationship'.
} 
association varied considerably. Some studies found that the relation between parent-child relationship and self-control was close to zero (e.g., Jones, Lynam, \& Piquero, 2015); some found the relation to be significant but small (e.g., Walters \& DeLisi, 2013), and some found the relation to be significant with medium effect size (e.g., Watt \& McNulty, 2016).

\section{The influence of self-control on parenting}

Adolescents are not passive recipients to parenting behaviors. Over the course of adolescence, their behaviors increasingly influence parenting behaviors. As such, adolescent development can be understood as a transactional or reciprocal process, where environmental factors (e.g., parenting, in this case) affect the development of adolescents (e.g., self-control) while adolescent's behavior can also evoke certain reactions from his/her environment (for details, see transactional model of development, Sameroff, 2010; social cognitive theory of personality, Bandura, 1999; genotype-environment correlation, Plomin, DeFries, \& Loehlin, 1977; ecological systems theory, Bronfenbrenner, 1979).

In parent-child dyads, parenting behaviors (e.g., parental trust and warmth) are related to parents' knowledge about the children (Kerr, Stattin, \& Trost, 1999). When parents know their children can exert self-control, resist temptations, and regulate their own behavior, parents are likely to trust their children, grant more autonomy and respond positively to their behavior (Buyukcan-Tetik, Finkenauer, Siersema, Vander Heyden \& Krabbendam, 2015). Prior studies involving adolescents and parents have found that adolescents with high levels of self-control elicit trust and warmth from their parents (Bradley \& Corwyn, 2007; Buyukcan-Tetik et al., 2015; Tiberio et al., 2016). However, longitudinal research conducted among adolescents also reveals non-significant effects of self-control on parenting over time 
(Eisenberg et al., 2005; Moilanen, Rasmussen, \& Padilla-Walker, 2015).

Similarly, children with good self-control are less impulsive and restless and engage in more socially desirable behavior, which may facilitate parents' relationship with them (Meldrum et al., 2012). Although some studies have supported this relation in adolescents (Bradley \& Corwyn, 2007; Otterpohl \& Wild, 2015), other studies failed to find such associations in adolescent samples (Meldrum et al., 2012). Conversely, adolescents with poor self-control are likely to engage in delinquent behavior and succumb to temptation, which may spur parents to assert their power by employing harsh or coercive practices to teach their children a lesson and ostensibly prevent the occurrence of the behavior in the future (Karreman et al., 2006; Kochanska, Aksan, \& Koenig, 1995; Silverman \& Ragusa, 1990). For instance, prior research found that adolescents with poor self-control are likely to elicit harsh parenting one year later (Brody \& Ge, 2001). However, inconsistent findings also emerge. To illustrate, Moilanen et al. (2015), found that adolescents' self-control negatively predicts mothers' but not fathers' authoritarian parenting.

\section{Potential moderators}

As shown above, the findings of the association of adolescent self-control and parenting are not consistent. This implies that the association between parenting and adolescent self-control may be moderated by a number of other factors, such as culture, age, and gender. A number of potential moderators are listed below.

\section{Theoretical moderators}

Type of parenting. Parenting is commonly categorized as positive and negative in terms of control and warmth (Darling \& Steinberg, 1993; O'Connor, 2002), suggesting that 
positive and negative parenting include both emotional (e.g., affection, warmth) and behavioral (e.g., monitoring and control) components. Close relationship refers to emotional bonding and thus it seems to solely represent the emotional component. Although a close emotional bonding is important to the development of self-control, it is not enough to instill children with self-control without consistent discipline and appropriate monitoring (Gottfredson \& Hirschi, 1990). This implies that the three categories of parenting may play a different role in the development of adolescent self-control. Therefore, in this study, we explored whether the three types of parenting differentially relate to self-control over the course of adolescence.

Age. As adolescents develop, they become more independent, they gain more autonomy from parents, negotiate more about social conventions, and become less attached to their family (Steinberg \& Silk, 2002), suggesting that the association of parenting and self-control is likely to decrease over the course of adolescence. Recent research examined the influence of parenting on effortful control from early childhood to early adolescence, revealing that the effects of parenting practices (both positive and negative practices) decreased as children grew older (Tiberio et al., 2016). Moreover, in Vazsonyi and Belliston's (2007) study, the associations between positive parenting (i.e., closeness, support, monitoring) and low self-control were lower among U.S. college students $(r=-.104,-.166$, and -.117, respectively) than those among U.S. urban ( $r=-.212,-.248$, and -.219 , respectively) and rural ( $r=-.185,-.326$, and -.132 , respectively) high school students. These findings, coinciding with the theories on autonomy development of adolescents (e.g., Collins \& Steinberg, 2006), led us to hypothesize that the association between parenting and self-control would diminish 
as adolescents become older.

Culture. Prior research revealed cross-cultural differences in parenting. For instance, Chinese parents are thought to use a more authoritarian style (or harsh parenting) than Western parents (Chao, 1994; Ng, Pomerantz, \& Deng, 2014). However, scholars argue that parenting is closely dependent on cultural contexts and therefore any type of parenting, no matter whether it is positive or negative, should be effective in socializing children in a given culture (Fu \& Markus, 2014; for on overview, see Smetana, 2017). Some cross-cultural studies directly compared the association between parenting and self-control in adolescents from different cultural and ethnical backgrounds, but yielded mixed evidence. For instance, research by Vazsonyi and colleagues found that the association between positive parenting (e.g., closeness and monitoring) was significantly related to self-control in Swiss, Dutch, Hungarian adolescents, but not in Slovenian and Japanese (for monitoring only) adolescents, and concluded such inconsistencies might be due to cultural differences in parenting (Vazsonyi \& Belliston, 2007; Vazsonyi, Trejos-Castillo, \& Huang, 2006). In Vazsonyi et al.'s (2016) study performed among Czech adolescents, the researchers found a positive relation between parental monitoring and self-control for Roma but not for non-Roma adolescents. Conversely, Li and colleagues (2015) found that the association between attachment to parents and self-control was largely invariant between Chinese and Italian adolescents. Given these findings, we explored whether culture moderates the "parenting - self-control" relation. Culture contains multiple dimensions and individualism is one of the most used variables to define cultures (Oyserman, Coon, \& Kemmelmeier, 2002). According to Hofstede's (2001), some countries are more individualistic than others and he developed an 
individualism index to reflect the levels of individualism of a country. In this sense, culture is treated as a continuum instead of a dichotomous category (e.g., Eastern vs. Western; or independent vs. dependent). Moreover, we were aware that a number of studies on self-control and parenting involved several ethnicities within their own country that may be not entirely mapped onto a country's levels of individualism (e.g., Asians residing in U.S.). Thus, we used both Hofstede's individualism score and ethnicity to capture the role of culture. In this study, we explored whether individualism score of the country where the samples were recruited and adolescent ethnicities would moderate the "parenting - self-control" association.

Adolescent gender. Research on gendered socialization (Gerson, 1985; Hagan, Simpson, \& Gillis, 1987; Hayslett-McCall \& Bernard, 2002) suggests that the causal processes of self-control may differ by gender. For instance, parents may tolerate certain behaviors (e.g., hang out with friends at night) by boys that would be quickly curtailed if displayed by girls. This suggests that the influence of parenting on self-control could differ between boys and girls. Some studies found that the associations between parenting and self-control were larger for girls than for boys (e.g., Evans, Simons, \& Simons, 2012; Larsen et al., 2012; Mandara \& Pikes, 2008). However, another line of work suggests that although parents may use different strategies to educate boys and girls, the effectiveness of parental socialization on children's development of self-control is comparable (Beaver, Wright, \& DeLisi, 2007; Chapple et al., 2010; Li et al., 2015; Lynskey, Winfree Jr., Esbensen, \& Clason, 2000). In this study, we explored whether the association between parenting and self-control differed as a function of proportions of boys and girls in the study sample. 
Parent gender. Traditionally mothers are considered to be the main caregiver in the home who provide daily care and are the most important socialization agents (Buist et al., 2002; Munroe, Munroe, Westling, \& Rosenberg, 1997; Song, Thompson, \& Ferrer, 2009). Yet, some theories suggest that fathers and mothers are equally important to the socialization of children (Gottfredson \& Hirschi, 1990; Lamb, 2010). Moreover, it is theorized that despite the traditional role of mothers in the family, fathers also play a significant role in children's adjustment, including the development of control of misbehaviors (Lamb \& Tamis-LeMonda, 2004). However, empirical evidence for these suggestions is mixed. In some studies, the association between parenting and self-control appears stronger for maternal parenting (Intravia, Jones, \& Piquero, 2012; Patock-Peckham, Cheong, Balhorn, \& Nagoshi, 2001) or for paternal parenting (Feldman \& Wentzel, 1990; Morris \& Age, 2009), whereas some studies find similar magnitude for both maternal and paternal parenting (e.g., Li et al., 2015; Nie et al., 2016; Özdemir et al., 2013). Given such disparities, we explored whether the "parenting - self-control" association varied as a function of the proportions of mothers and fathers in the study sample.

\section{Methodological moderators}

Reporting informant. Studies use a variety of methods to examine the relationship between parenting and self-control. Part of them utilizes self-report measures, part of them uses other informants (e.g., parent-report, teacher-report), and part of them even employs observational and behavioral methods. Results on the "parenting - self-control" association may vary across informants, because family members as well as teachers and observers may have different experiences or views regarding parent-child interactions and adolescent 
self-control (Duckworth \& Kern, 2011; Lanz, Scabini, Vermulst, \& Gerris, 2001). In the present study, we explored whether the relation between parenting and self-control was different among different reporting informants.

Consistency of reporting informant. We further examined whether consistency of reporting informant across constructs may moderate the link between parenting and self-control. Hypothetically, when the two constructs are assessed by the same informant (especially using self-report measures), their correlation is likely to be higher than when the two constructed are assessed by means of different informants (Willems et al., 2018).

Additionally, research found that the correlation between self-reports and other-reports on personality questionnaires is higher than the correlation between self-reports and behavioral tests (Duckworth \& Kern, 2011; Harden et al., 2017; Meyer, 2001). In light of this evidence, we explored whether the association between parenting and self-control is stronger when the two constructs were assessed using the same (i.e., consistent) rather than different (inconsistent) informants.

Study design. Both cross-sectional and longitudinal designs are used to test the association between self-control and parenting, but differences in the magnitude of concurrent versus longitudinal associations are not well quantified. Such comparisons have been done in other meta-analyses focusing on the link between self-control and deviance, with some studies revealing larger effect sizes for cross-sectional than for longitudinal study designs (Pratt \& Cullen, 2000) whereas others finding no significant differences between designs (Vazsonyi et al., 2017). In this meta-analysis, we tested whether the association between parenting and self-control would be different in magnitude for cross-sectional versus 
longitudinal studies. Using longitudinal studies, we also explored whether the influence of parenting on later self-control differed from the influence of adolescent self-control on later parenting. This examination, allowed us to pit the effect of parenting on self-control and the evocative effect of self-control on parenting against each other.

\section{Method}

The PRISMA checklist (Moher et al., 2015) was used as a guideline for the set-up of this meta-analysis. Furthermore, in order to facilitate transparency (Lakens, Hilgard \& Staaks, 2016), the aim and hypotheses of the present meta-analysis were pre-registered at AsPredicted ([website is omitted for masked review purpose]), and our full coding sheet including all the obtained effect sizes, moderating variables, and $\mathrm{R}$ analysis scripts is available online.

\section{Search of studies}

Articles were retrieved through computerized literature search of the electronic databases of the Education Resources Information Center (ERIC), PsychINFO, PubMed, and Web of Science. A literature search was conducted for studies published up to November 2016 with three categories of key phrases used for the search (1) key words containing variables concerning parenting or parent-adolescent relationship (parent* ${ }^{*}$ or mother $^{*}$ or father* $^{*}$ or parental or maternal* or attachment* or family* or bond*); (2) key words regarding self-control (self-control or self control or self-regulation or self regulation or self-discipline or self discipline or effortful-control or effortful control ${ }^{3}$; and (3) key words

\footnotetext{
${ }^{3}$ In the pre-registration, we did not include the search term effortful control because we expected our initial terms to yield articles on effortful control. However, during an initial trial of our search, we noticed that important effortful control articles were missing. As a result, we repeated the search including "effortful" and "control" as separate search terms to make our search more inclusive. Also, Prof. XXX and XXX [deliberately omitted for masked review purpose] were added as co-authors to the paper considering their invaluable insights and collaborations later in the project. Given that we included
} 
focusing on adolescents ${ }^{4}$ (adolescent* or adolescence or teen $*$ or youth $*$ or child* or student* or undergraduate or emerging adult* or young adult*).

\section{Inclusion criteria}

Studies were eligible for this meta-analysis when they met the following criteria. First, the study had to assess the relationship between any type of parenting (e.g., parental warmth, parental harshness) or parent-adolescent relationship (e.g., parental attachment, parental bond) and self-control. Specifically, the study had to report on self-control, or interrelated concepts such as self-regulation, effortful control, or domain-specific forms of control such as impulse regulation. In case no correlations were reported in the article, we contacted the corresponding author.

Second, the study had to focus on community-based samples, excluding clinical populations with psychological (e.g., cognitive impairments, autism) and/or physical symptoms (e.g., traumatic brain injury, diabetes, asthma), and/or criminal offenders. This is because we are interested in the general population; and clinical groups may influence the magnitude or direction of effect sizes (Rothbaum \& Weisz, 1994).

Third, the mean age of the participants in the study had to fall within the age range of adolescent period used in the current study (i.e., $10-22$ years). Twelve to eighteen is commonly seen as the age range of adolescence. However, we decided to broaden this range for two reasons. First, the beginning age of adolescence is related to puberty and WHO (2017) has considered that age 10 can be seen as the starting age of adolescence because of the earlier onset of puberty than before. Second, we consider age 22 as the upper bound of 
adolescence because in this new era youths have more time to develop instead of rushing for reproduction or making a living (National Research Council, 2015). Additionally, a recent study suggests that age 21 to 22 can be seen as the cut-off age when adolescents become adults in terms of brain maturity (Cohen et al., 2016). In the case of longitudinal designs, the study had to assess at least one of the constructs (i.e., parenting or self-control) during adolescence. For example, in a longitudinal study that assessed parental warmth and self-control at age 8 , age 12 , and age 14 , the concurrent and longitudinal correlations within and between age 12 and age 14 were included. Longitudinal correlations between age 8 and age 12 and between age 8 and age 14 were also included, but concurrent correlations at age 8 were excluded.

Fourth, the study had to be published in English in a peer-reviewed journal with full-text downloadable. We did not include unpublished work, review articles, book chapters, dissertations and conference abstracts as findings in these are often subsequently published in peer-reviewed journals. Inclusion of only peer-reviewed articles has been widely accepted in prior meta-analyses (e.g., Karreman et al., 2006; Lovejoy, Graczyk, O’Hare, \& Neuman, 2000; Slagt et al., 2016). Moreover, research has shown that meta-analyses that include unpublished studies are just as likely to find evidence for publication bias as those that do not (Ferguson \& Brannick, 2012).

\section{Selection procedure}

The initial search in the databases yielded 6,792 hits, after removing duplicates. The first two authors screened all abstracts independently, selecting articles for full text reading. This resulted in 814 potentially relevant articles. These articles were carefully screened to 
determine whether they met the inclusion criteria. A number of articles were excluded because: they did not include an appropriate measure of parenting and/or self-control $(k=$ $252)$; the study consisted of a clinical population or a population in the wrong age range $(k=$ 94); no full-text in English was available $(k=76)$; the article was not published as journal articles $(k=69)$; and no correlation table was available $(k=156)$. For the latter 156 articles, the corresponding authors were contacted by e-mail to request additional information. Some authors declined our invitation because they did not have access to the data anymore $(6 \%)$, some authors could not be contacted because no valid e-mail address was found (12\%), and some provided us with the necessary correlations $(15 \%$, yielding $k=24$ additional articles to include). Most of our e-mails remained unanswered (67\%). Finally, 191 studies met the selection criteria and were included in the meta-analysis. See Figure 1 for the PRISMA flowchart depicting the full search and inclusion process.

\section{Coding of the studies.}

We developed a detailed coding scheme based on guidelines proposed by Lipsey and Wilson (2001), to record study descriptors and study characteristics potentially moderating the relation between parenting and self-control in adolescence. Study descriptors included basic information for all studies, such as author names, year of publication, article title, details on data collection, and sample size. Study characteristics possibly moderating the relation between parenting and self-control in adolescence were grouped into two moderator categories: moderators of theoretical interest and methodological characteristics.

\section{Theoretical moderators}

Type of parenting. To assess possible moderating effect of parenting types, parenting 
practices and parenting characteristics were subdivided into the three categories (Davis et al., 2017; Doyle \& Mariewicz, 2005; Gallarin \& Alonso-Arbiol, 2012; Karavasilis et al., 2003; Hoeve et al., 2009; Karreman et al., 2006; Slagt et al., 2016): 1) positive parenting, including supervision, support, autonomy sensitivity, involvement, monitoring, authoritative parenting, warmth, positive expression etc.; 2) negative parenting, including harsh parenting, cold parenting, neglect, rejection, negative expressions, authoritarian, permissive, etc.; and 3) parent-child relationships, including social bonds, closeness, attachment, security, etc.. Studies were coded accordingly (coded categorically $1=$ positive parenting, $2=$ negative parenting, 3 = relationship). See supplemental table 1 for a detailed overview of the subdivision of parenting types.

Age. We coded age continuously. Some studies did not report age, but school grade. If this was the case, we took the average age of that grade. For example, in grade six in the USA children are on average between age 11-12, and we therefore considered 11.5 years as a mean age for this sample.

Culture. As mentioned above, we used individualism index and ethnicity to represent culture. We coded the level of individualism of the country where the data were collected according to Hofstede's individualism score (see www.hofstede-insights.com). The score is a continuous index with higher scores for more individualistic societies (e.g., an index of 91 for the USA) and lower scores for more collectivistic societies (e.g., an index of 17 for Taiwan).Regarding ethnicity, we coded ethnicity of adolescents (coded categorically, 1 $=$ balanced (i.e., no ethnicity exceeded $60 \%$ of the sample), 2 the sample consisted of more than $60 \%$ Caucasian, $3=$ the sample consisted of more than $60 \%$ African or 
African-American, $4=$ the sample consisted of more than $60 \%$ Asian or Asian American, $5=$ the sample consisted of more than $60 \%$ Hispanic, $6=$ other).

Adolescent gender. We coded adolescent gender according to the proportion of boys and girls included in the sample (coded categorically, $1=$ overall balanced (the percentage of boys or girls of the sample ranging between $40 \%$ and $60 \%$ ), $2=>60 \%$ boys, $3=>60 \%$ girls).

Parent gender. Studies were coded as to whether the parenting referred specifically to adolescents' mothers or to adolescents' fathers. Often, however, parenting measures assessed 'parenting' in general, and did not specifically mention whether the measure focused on mothers, fathers, or both parents. As a result, we categorized the variable as follows: $1=$ greater proportion of mothers ( $>60 \%$ of the sample), $2=$ greater proportion of fathers $(>60 \%$ of the sample), 3 = both parents, no clear proportion.

\section{Methodological characteristics}

Reporting informant. Studies were coded for the informant of the parenting measure (coded categorically, 1 = adolescent self-report, 2 = other-report, such as parent, mother, father, etc., 3 = observation, 4 = composite, combining measures of multiple informants or assessment modalities), for the informant of the self-control measure (coded categorically, 1 = adolescent self-report, 2 = other-report, such as parent, mother, father, etc., 3 =observation, 4 = composite, combining measures of multiple informants or assessment modalities).

Consistency reporting informant. Based on more detailed information of the informants, we coded whether the parenting and self-control measures were assessed by the same informant to assess reporting informant consistency (coded categorically, $1=$ consistent, 
$2=$ inconsistent $)$. Attention was paid to the elements that comprised of the composite score.

For example, if parenting and self-control were both composite scores combined from self-report and mother-report, then we considered the informant as consistent. If one composite score was combined from self-report and mother-report whereas the other combined self-report and teacher-report, we considered the informant as inconsistent.

Study design. Study design was coded as a categorical variable, including whether the effect size between parenting and self-control was derived from cross-sectional or longitudinal studies $(1$ = cross-sectional, 2 = longitudinal $)$. Additionally, for longitudinal studies we included effect sizes where parenting was measured first, and self-control was measured some time later (Parenting $(\mathrm{P}) \rightarrow$ Self-control (SC)). Similarly, we included effect sizes where self-control was measured first, and parenting was measured some time later (Self-control (SC) $\rightarrow$ Parenting $(\mathrm{P})$ ). As a result, we coded whether the effect size referred to the influence of parenting on self-control or the influence of self-control on parenting (coded categorically, 1 = from parenting to self-control, $P \rightarrow S C, 2=$ from self-control to parenting, $S C \rightarrow P)$.

\section{Inter-rater agreement}

Of the 814 eligible articles, $20 \%$ were randomly selected to be double coded by the first two authors. Intra-class correlation (for continuous variables) and Cohen's Kappa (for categorical variables) were calculated. Intra-class correlations for continuous variables were high, ranging from 0.78 (for age) to 0.99 (for sample size). Cohen's Kappa for the categorical ranged from 0.91 (for including/excluding studies) to 1.00 (for individualism score, study design, effect size direction, and informant parenting). To resolve disagreement, in-depth 
reading and discussion followed based on the content of the article. Together, these results showed good inter-rater reliability reflecting a good agreement for the study characteristics between the two independent raters.

\section{Effect sizes}

In order to investigate the magnitude of the relationship between parenting and self-control, Pearson's correlation coefficients $r$ s were obtained for all included studies (Lipsey \& Wilson, 2001). Zero-order correlation coefficients are bivariate estimates typically obtained from each empirical study's correlation matrix, or requested from authors if none was provided in the full-text. To ensure similar direction of effects, effect sizes were recoded if 1) parenting pertained to negative dimensions, and 2) self-control was measured using a scale of 'lack of self-control', or 'low self-control'. We used Fisher's $r$ to $z$ transformation, converting the effect size estimation from each association into an $E S_{Z}$ score to correct for skewness in the sampling distribution of $r$ (Lipsey \& Wilson, 2001). This $E S_{Z}$ scores is assumed to approach normality, which is necessary for the accurate determination of mean effect size estimates and for unbiased tests of statistical significance. As a result, $E S_{Z}$ were included in the analyses, and transformed back to Pearson's $r$ for interpretation purposes (Field, 2001; Lipsey \& Wilson, 2001) $)^{5}$. For moderator analyses, categorical variable categories were transformed to $k$-1 dummy variables through binary coding (Assink \& Wibbelink, 2016; Lipsey \& Wilson, 2001).

\footnotetext{
5 The Fisher's transformation of $r$ was done using the following formula: $\mathrm{ES}_{\mathrm{Zr}}=\frac{1}{2} \log _{e}\left[\frac{1+r}{1-r}\right]$. Any $\mathrm{ES}_{\mathrm{Zr}}$ can be transformed back into standard correlation form using the inverse of the ESZr transformation using the following formula: $r=\frac{e^{2 \mathrm{ESZr}}-1}{e^{2 \mathrm{ESZr}}+1}$ (see Field, 2001; Lipsey \& Wilson, 2001).
} 


\section{Publication Bias}

Previous research consistently showed that non-significant studies are more likely to be rejected for publication, or remain unsubmitted by authors (Torgerson, 2006; Lipsey \& Wilson, 2001). This publication bias may result in inflated effect sizes and restricted range of values in meta-analyses (Rosenthal, 1979). Therefore, it is important to statistically assess the possible influence of publication bias, before interpreting the final results. In the present study, we handled this problem by applying a funnel plot, plotting the distribution of each individual study's effect size on the horizontal axis against its precision expressed in standard errors on the vertical axis (Torgerson, 2006). If a publication bias affects the data, an asymmetrical funnel plot is to be expected (Begg, 1994). Additionally, Egger's test was applied to test the significance of the asymmetry of the plot, providing more precise information on the possible presence of publication bias (Egger, Smith, Schneider, \& Minder, 1997). When this test yields significant results, sensitivity analyses were conducted by applying the trim and fill method, correcting for the asymmetric plots by imputing missing effect sizes through a number of iterations (Duval \& Tweedie, 2000a, 2000b). Imputing non-existing effect sizes is, however, controversial and effect sizes produced by the trim and fill analyses should be interpreted with caution (Sutton, Duval, Tweedie, Abrams, \& Jones, 2000).

\section{Data analyses}

All analyses were conducted in the Metafor package in the open-source statistical R environment version 3.4.2 (Viechtbauer, 2010; R Core Team, 2017). Most studies reported on multiple effect sizes. For example, some studies included longitudinal data (yielding effect 
sizes for different time points), different raters (resulting in effect sizes separately for mother and father report), and effect sizes separately for boys and girls. It is likely that these effect sizes from the same study are more similar than effect sizes from different studies, because they rely on the same sample, data collection and sampling methods. When using nested effect sizes, however, the assumption of traditional meta-analyses that observations are independent and error terms are uncorrelated is violated (Lipsy \& Wilson, 2001; Rosenthal, 1984). Not taking into account this dependency can result in a biased result, as it may create artificially narrow confidence intervals and shrunken standard errors favoring statistical significance (Hox, Moerbeek \& Van de Schoot, 2010).

Strategies applied to handle the aforesaid dependency problem include selecting one effect size from each study, averaging effect sizes within studies or simply ignoring the dependency of effect sizes (Lipsey \& Wilson, 2001). However, more recently multilevel meta-analysis has been suggested as a more preferable tool, as it takes into account dependency while including all available effect sizes resulting in maximum information and greater statistical power (Assink \& Wibbelink, 2016; Hendriks et al., 2017; Hox et al., 2010; Van den Noortgate, López-Lopez, Marín-Martínez, \& Sánchez-Meca, 2013). Three level models apply when groups are nested within clusters, and thus are not independent from one another. In our case, we have variance at the effect size level (level 1) that are nested within a sample (level 2, e.g. effect sizes based on Add health data), with also variance between studies (level 3, taking into account effect sizes to vary between studies). Incorrectly modelling this dependency in the data will likely result in biased standard errors, making incorrect inferences about the relationships being studied (Viechtbauer, 2013). 
Accordingly, we applied a three-level model to account for the three sources of variance: (1) level 1 takes into account sampling variance of the effect sizes, (2) level 2 takes into account variance between effect sizes from the same sample, allowing effect sizes to vary within studies, (3) level 3 takes into account variance between studies, allowing effect sizes to vary between studies (Hox et al., 2010; Van den Noortgate et al., 2013). Additionally, since parameter estimates from different levels of analyses are not independent in this multilevel approach, no greater weight will be placed on studies with more sample sizes. Thus, a study that, for example, includes 10 effect sizes will not contribute 10 times more to the mean effect size compared to those only has one effect size (Van den Noortgate et al., 2013). Overall, multilevel modeling allows including effect sizes based on the same sample, providing more precision in estimating mean effect sizes while simultaneously modeling the nestedness of the data (Cheung, 2014; Van den Noortgate et al., 2013).

The current three-level analysis was conducted in three stages. First, the overall mean effect sizes were estimated to assess the strength of the association between parenting and self-control in adolescence. Second, we applied a likelihood ratio test to assess between-study and within-study heterogeneity. Important to note is that the level of 'study' entails dataset. For example, multiple papers are based on the Add Health dataset (http://www.cpc.unc.edu/ projects/addhealth). Accordingly, for the multilevel analyses we gave studies using the same dataset (e.g. all published studies using Add Health data) the same 'study ID', clustering them as if they were all from one published study. This allowed us to take into account this dependency, referring to the included studies as number of independent studies.

Third, if there was evidence for heterogeneity in effect sizes (presented as a $Q_{E}$ which, 
when significant, indicates heterogeneity among effect sizes), moderation analyses were conducted for moderators of theoretical interest and methodological characteristics. In order to obtain reliable results, moderator analyses were only conducted if each category contained at least five studies (parameter estimates are poor when number of studies is very small, Weiss et al., 2017).

The three-level analyses were conducted according to the three-level random effects model guidelines formulated by Wibbelink and Assink (2016), using the restricted maximum likelihood procedure for parameter estimation, and performed with the Metafor package in the open-source statistical software R version 3.4.2 R environment (Viechtbauer, 2010; R Core Team, 2017). Moreover, we used G*Power (version 3.1.9.2, Faul, Erdfelder, Lang, \& Buchner, 2007) to calculate the sample size needed for future research to obtain the mean correlation found in this research. Sample sizes for obtaining four levels of power (i.e., $.80, .90, .95, \& .99)$ with the alpha level of .05 were recommended.

\section{Results}

\section{Descriptive statistics}

The present meta-analysis included 191 articles, reporting on 159 independent studies and comprising of 1,540 effect sizes. All studies included in the meta-analysis are marked with an asterisk in the reference section. The overall sample size ${ }^{6}$ is $N=164,459$, with sample sizes of studies ranging between $N=47$ (Samuelson, Krueger, \& Wilson, 2012) and $N=$ 19,810 (Barnes \& Morris, 2012), and a mean age of 13.92 years. Publication year of the included studies ranged between 1990 and 2016, with the number of studies published

\footnotetext{
${ }^{6}$ The overall sample size was calculated by summing the largest sample size within unique samples when more than one effect size was collected.
} 
annually represented in Figure 2. Most studies included both boys and girls (80\%), with some studies focusing specifically on boys (7\%), some specifically focusing on girls (9\%), and some studies not providing gender descriptives (4\%). Some studies focused on parenting in general, without differentiation between mothers and fathers (44\%), some specifically focused on maternal parenting (40\%), and few studies specifically focused on paternal parenting (16\%). Of all included effect sizes, $53 \%$ focused on positive parenting, $33 \%$ on negative parenting, and $14 \%$ on parent-child relationships (see supplemental table 1 for details on parenting dimensions).

Studies were conducted worldwide, including studies from Australia, Canada, China, India, Mexico, with most effect sizes retrieved from the USA (62\%), South-Korea (10\%), Switzerland (6\%), and The Netherlands (4\%) (see Figure 3 for a graphical representation of the countries represented in the present meta-analysis). Hofstede's individualism indices ranged between 17 (Taiwan) / 18 (South-Korea), and 90 (Australia) / 91 (USA), with $k=28$ independent studies reporting on countries with an individualism score below 50 and $k=129$ studies reporting on countries with an individualism score above 50 .

The present meta-analysis included both cross-sectional (57\%) and longitudinal (43\%) effect sizes. Of the longitudinal effects, $56 \%$ measured parenting first and self-control some time later $(\mathrm{P} \rightarrow \mathrm{SC})$, and $44 \%$ measured self-control first and parenting some time later $(\mathrm{SC} \rightarrow \mathrm{P})$. Thus, the present meta-analysis included: 1) cross-sectional effect sizes 2) longitudinal $\mathrm{P} \rightarrow \mathrm{SC}$ effect sizes, where parenting predicted subsequent self-control and, 3) longitudinal SC $\rightarrow \mathrm{P}$, where adolescent self-control predicted subsequent parenting. These three groups describe different patterns of effects and should be treated individually. 
Therefore, we assessed overall effects of these three groups separately followed by statistical analyses testing whether these effects statistically differed. Of the 1,540 effect sizes, 876 concerned cross-sectional associations, 373 concerned longitudinal P $\rightarrow$ SC associations, and 291 concerned longitudinal SC $\rightarrow \mathrm{P}$ associations, respectively.

\section{Overall effects}

Cross-sectional association. The overall effect size of cross-sectional studies was statistically significant $\left(E S_{Z}=0.207\right.$, S.E. $=0.010, t=20.165, p<.001,95 \% \mathrm{CI}=[0.187$, $0.227])$, with substantial heterogeneity $\left(Q_{E}(875)=13140.584, p<.001\right)$. Using inverse version of the Fisher's (1921) $r$-to- $z$ formula, we transformed this effect size back to Pearson $r$ for interpretation purposes. We found that the cross-sectional association between parenting and self-control in adolescence was $r=.204$.

Longitudinal associations: parenting $\rightarrow$ self-control. The overall effect size of longitudinal $\mathrm{P} \rightarrow \mathrm{SC}$ was also statistically significant $\left(E S_{Z}=0.158\right.$, S.E. $=0.015, t=10.238$, $p<.001,95 \% \mathrm{CI}=[0.128,0.188])$, with substantial heterogeneity $\left(Q_{E}(372)=3349.047, p\right.$ $<.001)$. We found that the longitudinal association where parenting was measured first and self-control later was $r=.157$.

Longitudinal associations: self-control $\rightarrow$ parenting. For longitudinal SC $\rightarrow$, the overall effect size was also statistically significant $\left(E S_{Z}=0.156\right.$, S.E. $0.022, t=7.123, p$ $<.001,95 \% \mathrm{CI}=[0.113,0.199]$, with substantial heterogeneity $\left(Q_{E}(290)=2293.718, p\right.$ $<.001)$. The longitudinal association where self-control was measured first and parenting later was $r=.155$.

Comparison between cross-sectional and longitudinal effect sizes. As reported 
above, there were some observed differences in the effect sizes between cross-sectional and longitudinal studies, and therefore we tested whether this difference was statistically significant. Applying three-level analyses, we found an overall significant difference between the three groups $F(2,1,537)=26.14, p<.001$, and significant heterogeneity in effect sizes $Q_{E}(1537)=1,878.35, p<.001$. Further slope analyses revealed associations for longitudinal P $\rightarrow \mathrm{SC}$ to be significantly smaller than cross-sectional associations $(\beta=-.050, S . E .=0.010, t$ $=-6.228, p<.00195 \% \mathrm{CI}=[-.066,-.034])$. Similarly, associations for longitudinal $\mathrm{SC} \rightarrow \mathrm{P}$ were also significantly smaller than cross-sectional associations $(\beta=-.052$, S.E. $=0.010, t=$ $-6.058, p<.00195 \% \mathrm{CI}=[-.068,-.035])$. Results showed no significant difference in the associations between longitudinal $\mathrm{P} \rightarrow \mathrm{SC}$ and $\mathrm{SC} \rightarrow \mathrm{P}(\beta=-.002, S . E .=0.010, t=-0.174, p$ $=.862,95 \% \mathrm{CI}=[-.019, .016])($ see Table 1$)$.

Subsequently, analyses for these three groups were conducted separately. First, a random-effect model was utilized to calculate variance at the sampling, within-study, and between-study levels. Second, multiple potential moderators were tested individually. Third, a multiple-moderator model including all the significant moderators was performed to control for the associations among moderators. Finally, we also checked publication bias using the Funnel plot (Egger et al., 1997).

\section{Cross-sectional studies}

Variance of the overall effect size. The variance at the within-study level (estimate $=.010, p<.001)$ and the between-study level (estimate $=.011, p<.001)$ were both significant. Follow-up analyses found that variance at the sampling, within-study, and between-study level was 5.13\%, 45.11\%, and 49.76\%, respectively. Hunter and Schmidt 
(1990) proposed that heterogeneity can be considered as substantial, if less than $75 \%$ of the variance can be attributed to the sampling variance and that in this case examination of the moderating effects of the study and/or effect size characteristics on the overall effect can be fruitful. In our study, only $5.13 \%$ variance was explained by the samples, indicating that continuous exploration of potential moderators is meaningful.

Moderator analyses. Considering the large statistical power (is $N=164,459$ ), we were confident to assess potential moderators of theoretical and methodological interest (see Table 2). Regarding the moderators of theoretical interests, the only significant moderator was type of parenting $\left(Q_{E}(873)=12763.278, p<.001\right.$; Omnibus test: $\left.F(2,873)=3.483, p=.031\right)$. None of the other moderators of theoretical interest, including cultural characteristics (ethnicity, Hofstede's individualism), parent gender (i.e., whether the effect refers to mother or father), adolescent gender (i.e., whether the effect refers to boys and girls), or age of adolescents $^{7}$ was significant.

Pertaining to the moderators of methodological interest, all the three moderators in this category were significant: informant of parenting measure $\left(Q_{E}(871)=13034.229, p\right.$ $<.001$; Omnibus test: $F(3,871)=5.172, p=.002)$, informant of self-control measure $\left(Q_{E}(872)=12859.771, p<.001 ;\right.$ Omnibus test: $\left.F(3,872)=5.068, p=.002\right)$, and consistency of the informant of the parenting and self-control measures $\left(Q_{E}(873)=13104.211, p<.001\right.$; Omnibus test: $F(1,874)=15.043, p<.001)$.

Significant moderators. Based on the significant moderators found above, follow-up

\footnotetext{
7 We were also interested to see whether age could show a non-linear pattern. To this end, we squared and cubic age and checked whether the squared/cubic age served as a significant moderator. However, our results showed no significant quadratic $\left(Q_{\mathrm{E}}(830)=10384.754, p<.001\right.$; Omnibus $\left.F(1,830)=1.698, p=.193\right)$ or the cubic pattern $\left(Q_{\mathrm{E}}(830)=10361.892\right.$ $p<.001$; Omnibus $F(1,830)=1.693, p=.194)$.
} 
comparison was conducted and the results are summarized in Table 3. Regarding the type of parenting, we found that the effect sizes for the "positive parenting - self-control" $\left(E S_{Z}=.213\right.$, S.E. $=0.011, t=18.826, p<.001,95 \% \mathrm{CI}=[0.191,0.235], r=.210)$, "negative parenting self-control" $\left(E S_{Z}=0.188, S . E .=0.013, t=14.864, p<.001,95 \% \mathrm{CI}=[0.163,0.212], r\right.$ $=.186)$ and "parent-child relationship - self-control" associations were all significant $\left(E S_{Z}=\right.$ 0.220$, S.E. $=0.016, t=13.676, p<.001,95 \% \mathrm{CI}=[0.188,0.252], r=.217)$. Results of further comparison suggested that the relationship for negative parenting was significantly smaller than that for positive parenting (see Table 3).

Regarding the informant of parenting measure, we found that the effect sizes of the relationship between parenting and self-control were all significant when parenting was measured using adolescent self-report $\left(E S_{Z}=0.206, S . E .=0.011, t=19.041, p<.001,95 \%\right.$ $\mathrm{CI}=[0.185,0.228], r=.203)$, other-report $\left(E S_{Z}=0.199, S . E .=0.016, t=12.264, p<.001\right.$, $95 \% \mathrm{CI}=[0.167,0.231], r=.196)$, observation $\left(E S_{Z}=0.114\right.$, S.E. $=0.032, t=3.574, p$ $<.001,95 \% \mathrm{CI}=[0.051,0.176], r=.114)$, and composite measures $\left(E S_{Z}=0.276\right.$, S.E. $=$ $0.003, t=9.060, p<.001,95 \% \mathrm{CI}=[0.216,0.336], r=.269)$. Results of further comparison indicated that effect sizes for studies using composite measures were significantly larger than those using self-report, other-report, and observation, and that effect sizes for studies using observation measure to assess parenting were significantly lower than those using adolescent self-report and other-report (see Table 3).

With respect to the informant of self-control measures, we found that effect sizes of the relationship between parenting and self-control were all significant when self-control was assessed using adolescent self-report $\left(E S_{Z}=0.203, S . E .=0.011, t=18.667, p<.001,95 \% \mathrm{CI}\right.$ 
$=[0.182,0.224], r=.200)$, other-report $\left(E S_{Z}=0.188, S . E .=0.017, t=11.161, p<.001,95 \%\right.$

$\mathrm{CI}=[0.155,0.221], r=.186)$, observation $\left(E S_{Z}=0.121\right.$, S.E. $=0.039, t=3.092, p=.002,95 \%$

$\mathrm{CI}=[0.044,0.198], r=.120)$, and composite measures $\left(E S_{Z}=0.297, S . E .=0.029, t=\right.$ $10.291, p<.001,95 \% \mathrm{CI}=[0.240,0.353], r=.289)$. Follow-up comparison indicated that effect sizes of studies using composite measures to assess self-control were significantly larger than those using adolescent self-report, other-report, and observation, and that effect sizes of studies using observation measures to assess self-control were significantly lower than those using adolescent self-report (see Table 3).

For the consistency of the reporting informant on parenting and self-control measures, effect sizes were both significant for studies using consistent $\left(E S_{Z}=0.223\right.$, S.E. $=0.011, t=$ $19.785, p<.001,95 \% \mathrm{CI}=[0.201,0.245], r=.219)$ and inconsistent report informant $\left(E S_{Z}=\right.$ 0.168$, S.E. $=0.015, t=11.475, p<.001,95 \% \mathrm{CI}=[0.139,0.196], r=.166)$. Results of follow-up comparison showed that effect sizes of studies using inconsistent reporting informants were significantly lower than those using consistent reporting informants (see Table 3).

Multiple moderator model. According to Hox (2010), moderators may be interrelated, possibly causing multicollinearity problems in the analyses. To overcome this, a multiple moderator model which included all significant moderators found in the individual moderation test above was performed. The results of this model are summarized in Table 4. Omnibus test showed a significant results $(F(9,865)=6.157, p<.001)$, suggesting that at least one of the regression coefficients of the moderators significantly deviated from zero. These results indicated that negative parenting (vs. positive parenting), composite measure of 
self-control (vs. adolescent self-report), and inconsistent report informant (vs. consistent report informant) had unique moderating effects on the relationship between parenting and self-control.

Publication bias. Considering our large sample size, and that, for numerous studies, the association between parenting and self-control was not the primary research interest, we assumed little influence of publication bias. To statistically check this assumption, we inspected funnel plot using Fisher's $z$ transformations (see Figure 4a.), and applied Egger's regression test (Egger et al., 1997; Torgerson, 2006). Results of regression test for Funnel plot asymmetry found that there was no significant asymmetry, $z=-1.506, p=.132$, suggesting that no significant publication bias was detected for the results found above.

\section{Longitudinal $\mathrm{P} \rightarrow \mathrm{SC}$ studies}

Variance of the overall effect size. The variance at the within-study level (estimate $=.006, p<.001)$ and the between-study level (estimate $=.007, p<.001)$ were both significant. Follow-up analyses found that variance at the sampling, within-study, and between-study level was $7.01 \%, 44.29 \%$, and $48.69 \%$, respectively, indicating that continued exploration of potential moderators is meaningful.

Moderator analyses. Similar to cross-sectional analyses, moderators of theoretical and methodological interest were tested in the longitudinal data and are summarized in Table 5. Moderator analyses were only conducted if each category contained at least five studies (parameter estimates are poor when number of studies is very small). As a result, we could not test the moderating effect of adolescent gender, and reporting informant of self-control. Regarding the moderators of theoretical interest, the only significant moderator was parent 
gender, $Q_{E}(366)=3188.95, p<.001$; Omnibus test: $F(2,366)=6.150, p=.002$. None of the other moderators of theoretical interest, including type of parenting (i.e., positive / negative / relationship), or cultural characteristics (ethnicity, Hofstede's individualism), was significant.

Pertaining to the moderators of methodological interest, we tested informant of parenting measure and consistency of informants. Both informant of parenting measure $\left(Q_{E}\right.$ $(360)=3132.17, p<.001$, Omnibus test: $F(3,360)=3.770, p=0.011)$, and consistency of informants $\left(Q_{E}(371)=3186.39\right.$, Omnibus test: $\left.F(1,371)=6.562, p=0.011\right)$, yielded significant results.

Significant moderators. Follow-up analyses were conducted to analyze the aforementioned significant moderators with results presented in Table 6. Regarding parent gender, we found significant associations for "maternal parenting - self-control" $\left(E S_{Z}=0.194\right.$, S.E. $=0.018, t=10.967, p=0.002,95 \% \mathrm{CI}=[0.159,0.229], r=.192)$, "paternal parenting self-control" $\left(E S_{Z}=0.181, S . E .=0.023, t=7.800, p<0.001,95 \% \mathrm{CI}=[0.135,0.227], r\right.$ $=.178)$, and "both parents parenting - self-control" $\left(E S_{Z}=0.129, S . E .=0.017, t=7.590, p=\right.$ $<.001,95 \% \mathrm{CI}=[0.096,0.163], r=.129)$. Results of further comparison suggested that when children were asked to rate parenting for 'both parents', the association was significantly smaller than when asked specifically for maternal parenting or paternal parenting, respectively. The association between parenting and self-control was not significantly different for mothers and fathers (see Table 6.)

With respect to the reporting informant of parenting measures, we found that effect sizes of the relationship between parenting and self-control were all significant when parenting was assessed using adolescent self-report $\left(E S_{Z}=0.152, S . E .=0.018, t=8.554, p\right.$ 
$<.001,95 \% \mathrm{CI}=[0.117,0.187], r=.151)$, other-report $\left(E S_{Z}=0.150, S . E .=0.021, t=7.013\right.$, $p<.001,95 \% \mathrm{CI}=[0.108,0.192], r=.149)$, observation $\left(E S_{Z}=0.110, S . E .=0.030, t=3.54\right.$, $p<.001,95 \% \mathrm{CI}=[0.169,0.266], r=.110)$, and composite measures $\left(E S_{Z}=0.218, S . E .=\right.$ $0.025, t=8.881, p<.001,95 \% \mathrm{CI}=[0.169,0.266], r=.215)$. Follow-up comparison indicated that effect sizes of studies using composite measures to assess parenting were significantly larger than those using self-report, other-report, or observation.

For the consistency of the reporting informant of parenting and self-control measures, effect sizes were both significant for studies using consistent $\left(E S_{Z}=0.138, S . E .=0.017, t=\right.$ $8.259, p<.001,95 \% \mathrm{CI}=[0.105,0.170], r=.137)$ and inconsistent reporting informant $\left(E S_{Z}\right.$ $=0.179$, S.E. $=0.017, t=10.610, p<.001,95 \% \mathrm{CI}=[0.146,0.212], r=.177)$. Results of follow-up comparison showed that effect sizes of studies using inconsistent reporting informants were significantly larger than those using a consistent reporting informant (see Table 6).

Multiple moderator model. For the multiple moderator model, all significant moderators illustrated in Table 6 were included into one single model to test their robustness. The results suggested that at least one of the regression coefficients of the moderators significantly deviated from zero $(F(6,353)=4.278, p<.001)$. These results indicated that parent gender (both, vs father/mother), and inconsistent informant reports (vs. consistent) had unique moderating effects on the relationship between parenting and self-control (see Table 7).

Publication bias. The funnel plot is illustrated in Figure $4 \mathrm{~b}$, with results of the regression test suggesting significant asymmetry, $z=2.389, p=.017$. This suggests that there 
was indication of publication bias for longitudinal studies on the association between parenting and self-control. Subsequently, a trim and fill procedure was applied to take publication bias into account, resulting in an adjusted effect size of $E S_{z}=0.106$ (S.E. $=0.007$, 95\% CI [0.092, 0.119], $p<.001 r=.105)$.

\section{Longitudinal SC $\rightarrow$ P}

Variance of the overall effect size. The variance at the within-study level (estimate $=.005, p<.001)$ and the between-study level (estimate $=.007, p<.001)$ were both significant. Follow-up analyses found that variance at the sampling, within-study, and between-study level was $5.46 \%, 26.29 \%, 68.26 \%$ respectively, indicating that continuous exploration of potential moderators was meaningful.

Moderator analyses. Similar to longitudinal $\mathrm{P} \rightarrow \mathrm{SC}$ analyses, we could not test the moderating effect of adolescent gender, and informant on self-control because these categories did not contain enough studies. Regarding the moderators of theoretical interest, none of them was significant, including type of parenting (i.e., positive / negative / relationship), cultural characteristics (ethnicity, Hofstede's individualism), and parent gender (maternal parenting / paternal parenting / both parents) (see Table 8.).

Pertaining to the moderators of methodological interest, including reporting informant of parenting measure and consistency of informants, only reporting informant of parenting measure yielded significant results $\left(Q_{E}(286)=1999.973, p<.001\right.$; Omnibus test: $F(3,286)=$ $7.075, p<.001)$.

Significant moderators. Follow-up analyses were conducted to further analyze the significant moderators. With respect to the informant of parenting measure, we found that 
effect sizes of the relation between parenting and self-control were all significant when parenting was assessed using adolescent self-report $\left(E S_{Z}=0.154\right.$, S.E. $=0.024, t=6.478, p$ $<.001,95 \% \mathrm{CI}=[0.107,0.201], r=.152)$, other-report $\left(E S_{Z}=0.096, S . E .=0.026, t=3.636\right.$, $p<.001,95 \% \mathrm{CI}=[0.044,0.148], r=.095)$, observation $\left(E S_{Z}=0.113\right.$, S.E. $=0.034, t=$ $3.310, p<.001,95 \% \mathrm{CI}=[0.046,0.180], r=.113)$, and composite measures $\left(E S_{Z}=0.232, S . E\right.$. $=0.035, t=6.684, p<.001,95 \% \mathrm{CI}=[0.164,0.300], r=.228)$. Follow-up comparison indicated that effect sizes of studies using composite measures to assess parenting were significantly larger than those using self-report, other-report, or observation (see Table 9). No multiple moderator analyses were applied because only one moderator was significant.

Publication bias. Funnel plot (illustrated in Figure 4c.) and regression test indicated publication bias, $z=3.694, p<.001$. Accordingly, trim and fill procedure was applied to take publication bias into account, resulting in an adjusted effect size of $E S_{z}=0.153$ (S.E. $=0.007$, $95 \%$ CI [0.139, 0.167], $p<.001, r=.152)$.

\section{Discussion}

Parenting has long been considered important to the development of self-control (Gottfredson \& Hirschi, 1990; Eisenberg et al., 2003, 2005; Kochanska et al., 1995; Kopp, 1982; Sameroff, 2010). The current three-level meta-analysis is the first to synthesize the relation between broad types of parenting (i.e., positive parenting, negative parenting, and parent-adolescent relationship) and self-control of adolescents aged 10 to 22 years. Analyses were based on159 independent studies, 1540 effect sizes, from four continents, with a sample size of is $N=164,459$ providing large statistical power. Our results showed that: (1) parenting is concurrently and longitudinally associated with self-control throughout adolescence; (2) 
adolescent self-control significantly predicts subsequent parenting; and the predictive effect of parenting on self-control and the one of self-control on parenting show similar magnitude; and (3) the relations between parenting and self-control (for both directions) largely hold equal across cultures, ethnicities, parent and adolescent gender, and age, while the associations are moderated by a few methodological factors, such as reporting informant consistency. These findings provide a rich description of whether and how self-control and parenting are related across the entire period of adolescence.

Despite the changes that occur in adolescence such as eminent independence from parents and more investment in peer and romantic relationships (Connolly et al., 2004; Nickerson \& Nagle, 2005; Song et al., 2009), our results showed that parenting was related to self-control in adolescence both concurrently and longitudinally. Speaking to the robustness of our findings, the effect sizes for the "parent-child relationship - self-control" association were similar to those reported in a recent meta-analysis focusing on the attachment security status and its relation with effortful control in children and adolescents up to age 18 (Pallini et al., 2018).

Beyond the influence of parenting on self-control, our findings based on longitudinal studies also revealed a significant effect of adolescent self-control on subsequent parenting. Previous research has assumed an evocative effect from child's outcomes to parenting (e.g., Kochanska et al., 1995; Silverman \& Ragusa, 1990; Sameroff, 2010), but existing findings were inconsistent (e.g., Lee et al., 2012; Meldrum et al., 2012; Moilanen et al., 2015). The present findings, based on 291 effect sizes, provide support for the notion that adolescent self-control at a given time point does affect (or evoke) subsequent parenting behaviors, such 
that high self-control leads to more positive parenting, less negative parenting, and a positive parent-adolescent relationship. Low self-control, in contrast, evokes parents' diminished use of positive parenting (e.g., warmth, support) and increased use of negative parenting (e.g., physical and coercive punishment), and gives rise to a more negative parent-child relationship. We did not find a significant difference in the magnitude of the effect of parenting on adolescent self-control and the evocative effect (i.e., the effect of adolescent self-control on parenting), which is consistent with developmental theories underpinning the importance of bidirectional interactions between adolescents and their (parental) environment for adolescent development (Bandura, 1999; Bronfenbrenner, 1979; Sameroff, 2010; Plomin et al., 1977). The current results revealed that, throughout adolescence, parenting continues to affect the development of adolescent self-control and, adolescent self-control continues to affect parenting.

The associations tested were found to be moderated by a few-mainly methodological—factors (see summary in Table 10). However, moderators for cross-sectional studies did not necessarily extend to longitudinal studies (e.g., type of parenting) and vice versa (e.g., parent gender). Moreover, for some moderators, there was not enough information to detect their effects (e.g., adolescent gender for longitudinal studies). Some moderators (e.g., consistency of informants) even showed contradictory moderating effects for cross-sectional and longitudinal studies. For example, in cross-sectional studies effect sizes were larger for studies using consistent than inconsistent report informants, but in longitudinal studies ( $\mathrm{SC} \rightarrow \mathrm{P}$ ) effect sizes were larger for studies using inconsistent than consistent report informants. 
Based on cross-sectional studies, which comprised of more than half of the total effect sizes, we found that the relation between parenting and self-control tended to be stronger when parenting was positive than when parenting was negative, when self-control was measured using multiple report-informants compared to single report informant, and when informants of parenting and self-control were consistent.

Given the small moderating effects, our results suggest that the inconsistent findings regarding the association between parenting and self-control in the past literature may be largely due to methodological artifacts rather than theoretical misspecification. In addition, we found a publication bias in longitudinal but not in cross-sectional studies, which may be a reason why moderators between cross-sectional and longitudinal studies were not entirely consistent, because publication bias may also influence the estimates of between-study variance (Jackson, 2006).

\section{Theoretical implications}

The present results bear several theoretical implications. First, a number of theories and theoretical perspectives (e.g., attachment theory, the general theory of crime, the development of self-control and conscience; and the unified theory of development) propose that good parenting is a crucial source of self-control in children (Bowlby, 1969; Gottfredson \& Hirschi, 1990; Kochanska, 1993; Kopp, 1982; Sameroff, 2010). The current findings imply that this proposition extends to adolescence, and show that positive parenting and good parent-child relationships continue to play an essential role in shaping the development of self-control from early to late adolescence and emerging adulthood; conversely, negative parenting and poor parent-child relationship continue to hamper the development of 
adolescents' self-control. Second, the importance of parenting on adolescent self-control is largely equivalent across different cultures, ethnicities, and adolescent and parent gender. This suggests that the above mentioned theories and viewpoints regarding the influence of parenting on self-control are generally applicable across different demographic backgrounds, thus demonstrating their cross-cultural validity. Third, the aforesaid theories and perspectives mainly focus on parental effect on adolescents' self-control, and disregarding the examination of the evocative effect, as pointed out by some scholars (Lerner, 2002; Tiberio et al., 2016; Vazsonyi \& Huang, 2010). The current results demonstrated both parent- and child-effect regarding the relations between parenting and self-control, which suggests that the existing theories may need to take both parent and child effects into consideration to better capture the dynamic relation between parenting and self-control in adolescents. Last but not least, prior studies have found individual differences in the genetic and neural underpinnings of self-control (Beaver et al., 2007, 2008; Casey et al., 2008). Our findings may enhance our understanding about how these differences in genetic and neural underpinnings come from and their development over time.

\section{Limitations}

The present findings should be interpreted with caution. First, the sample size for the cross-sectional analyses was much larger than the sample size for the longitudinal analyses, resulting in more powerful analyses for the former. Due to this power issue, not all moderators could be tested in the longitudinal analyses. Second, our meta-analysis only included community-based adolescent samples, and its results may not be generalized to clinical samples (e.g., diagnosed with attentional disorder and hyperactivity disorder, autism 
spectrum disorder, diabetes) or samples with specific characteristics proposed to be related to self-control (e.g., prisoners, drug-addicts). Third, our research aim was to explore how much parenting may explain individual differences rather than changes in self-control, and thus baseline level of self-control was not taken into account. This issue is not without precedence (e.g., Davis et al., 2017; Karreman et al., 2006; Pallini et al., 2018; Vazsonyi et al., 2017). Nevertheless, future research that aims to explore how parenting affects the changes in self-control should consider to apply longitudinal cross-lagged model to examine this issue. Last, we found a significant publication bias in the longitudinal "parenting - self-control" associations, which implies that findings from these studies may be biased.

\section{Future direction}

The current study bears important implications for future research. First, the included studies came from several continents and the sample size was large (over 160,000). However, a majority of effect sizes (i.e., 62\%) were retrieved from studies conducted in the USA, and no or few eligible effect sizes were based on studies from African, South American, Southeastern Asian, Central Asian, and Eastern European countries. Language and research funding may explain this discrepancy. In order to further strengthen the current findings, we encourage more international studies to be conducted and published in English. Considering our data and scripts are freely accessible online, extending our results with international data is feasible. This provides opportunities for other scholars who have different theoretical preference to categorize parenting such as warmth / behavioral control / autonomy support (e.g., Prinzie, Stams, Deković, Reijntjes, \& Belsky, 2009) to analyze our data for different research questions and facilitates an update of the meta-analysis in the future. 
Second, among the studies included in this meta-analysis, many assessed parenting in general without separately referring to mothers or fathers. Although mothers' parenting and fathers' parenting often show medium-to-high correlations (e.g., Li et al., 2015; Nie et al., 2016; Özdemir et al., 2013), examining parenting for mothers and fathers separately would be promising. It may allow researchers to identify the similarities and differences between maternal and paternal influences on adolescent self-control. Future research regarding this issue may want to separately explore the effect of mothers and fathers.

Third, the effect sizes of parenting indicate that adolescent self-control is influenced by multiple factors and other socialization agents. Some studies have been conducted to address the effects of other socialization agents, such as peers or teachers (e.g., Alvarez-Rivera \& Fox, 2010; Meldrum, 2008; Turner, Piquero, \& Pratt, 2005). Additionally, recent research emphasizes the influence of genetic factors on individual differences in self-control (e.g., Beaver, Connolly, Schwartz, Al-Ghamdi, \& Kobeisy, 2013; Beaver et al., 2009; Willems et al., 2018), parenting (e.g., for a review, see Klahr \& Burt, 2014), or both (e.g., Beaver, Ferguson, \& Lynn-Whaley, 2010). However, contemporary research has focused largely on the role of parents on self-control rather than other etiological sources (reflected by the large number of studies included in this meta-analysis). Thus, much of the literature evaluates the development of self-control as a result of family socialization yet more evidence from other contextual and biological studies is necessary to paint a more complete picture of the development of self-control in adolescence.

Last, this meta-analysis provided overall effect sizes for cross-sectional and longitudinal associations between parenting and self-control. This knowledge on the average 
effect size allows us to provide additional recommendations for future research. Specifically, it provides information on the number of participants necessary to detect the current findings. Doing so will allow researchers to gauge the appropriate level of conservatism or liberalism they prefer when recruiting participants, and helps researchers to make the most of their time and resources. Table 11 summarizes sample sizes to achieve the correlation coefficients transformed back from effect sizes (i.e., $\mathrm{ES}_{r}$ ) at four levels of power with alpha level of .05. It should be noted that these sample sizes are estimated for bivariate correlations. If researchers wish to conduct other statistical analyses in future studies, they may need to recalculate the sample size based on the effect sizes found in this study. However, this can be easily implemented in $\mathrm{G}^{*}$ Power Software or using other approaches (e.g., Monte Carlo simulation).

\section{Conclusive remarks}

Ill decisions and reckless behaviors due to low self-control in adolescence are at the cost of individual physical and psychosocial functioning as well as social security both concurrently and longitudinally (Moffit et al., 2011; Caspi et al., 2017). The current study suggests that parenting significantly contributes to self-control in adolescents aged 10 to 22 . It also suggests that adolescent self-control shows a significant evocative effect on subsequent parenting. These relationships are largely equal across cultures, ethnicities, parent and child gender, and age of adolescents; and only a few (mainly methodological) factors moderate this relationship. Our findings provide further evidence for the importance of considering the continuous and dynamic interplay of the development of self-control and environment (parenting/parent-child relationship) across the adolescent period. 


\section{References}

References list is currently omitted in this pre-print, but will be made publicly available as soon as the article gets published. 


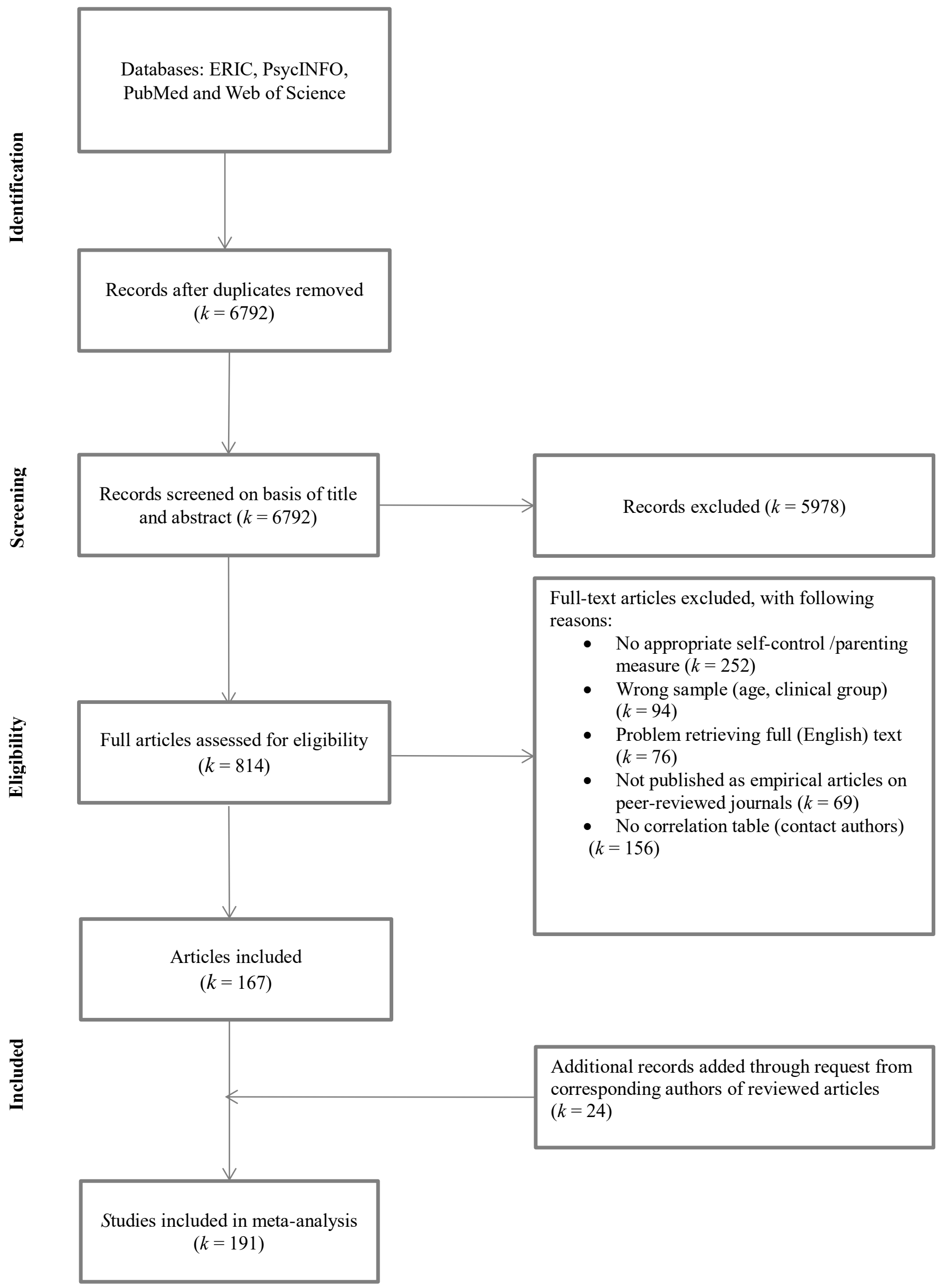

Figure 1. PRISMA flowchart used to identify studies for detailed analysis of parenting and self-control 


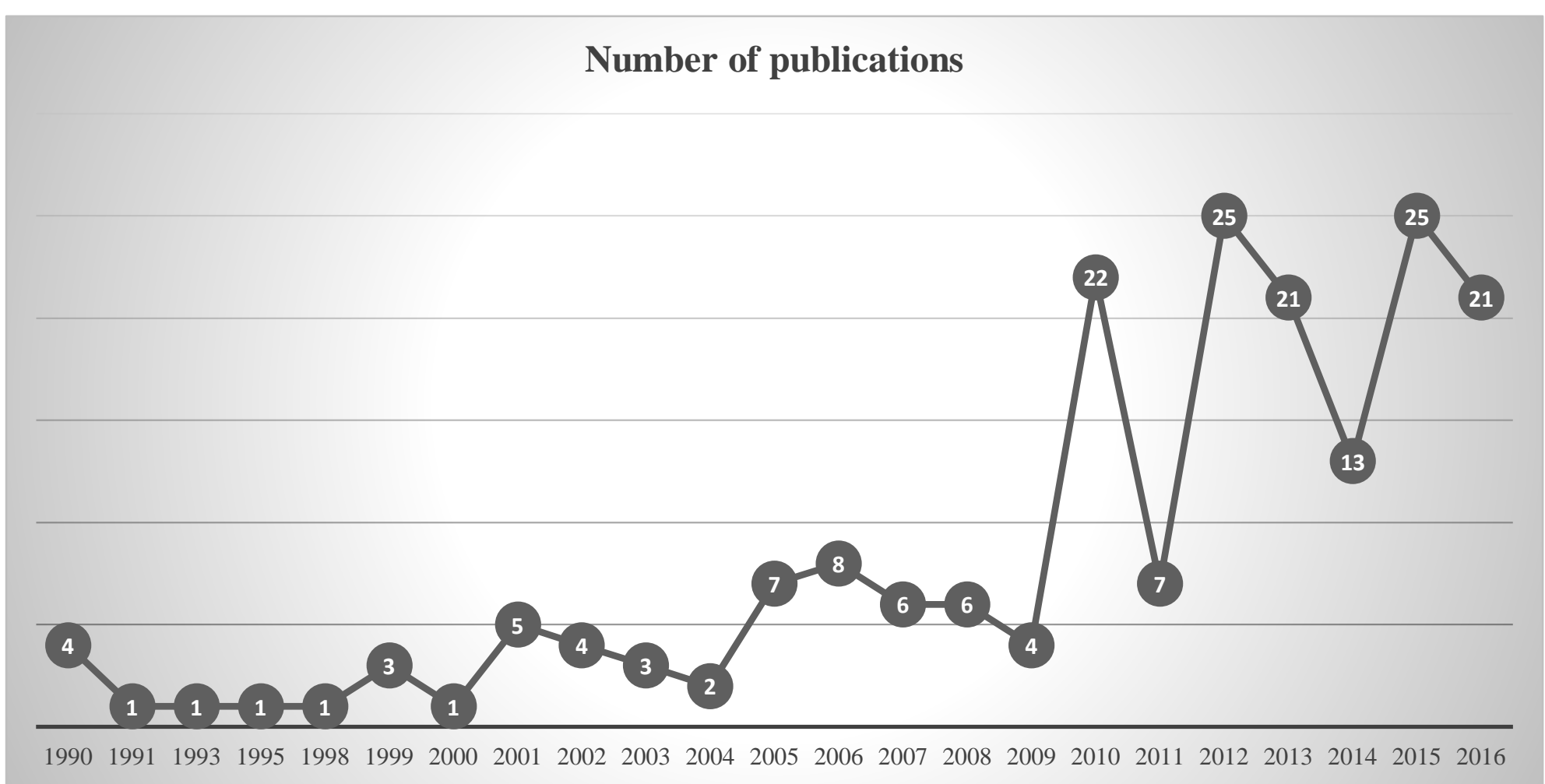

Figure 2. Number of included studies published annually 


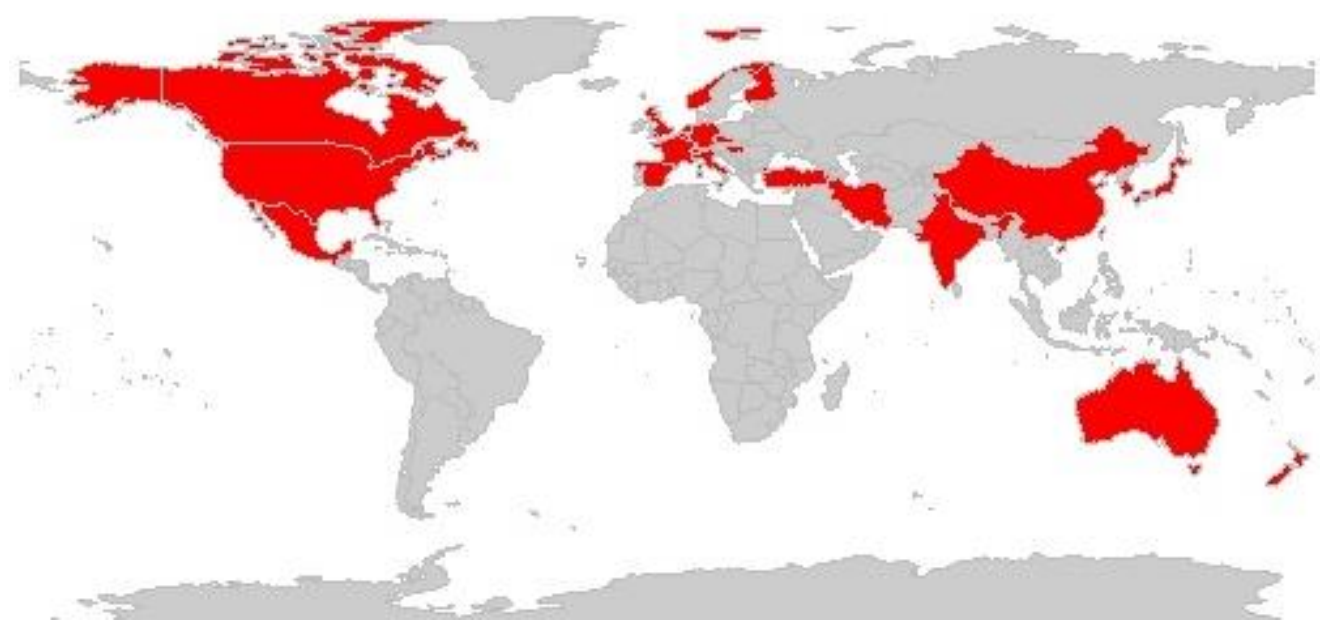

Figure 3. Graphical representation of countries represented in the meta-analysis 

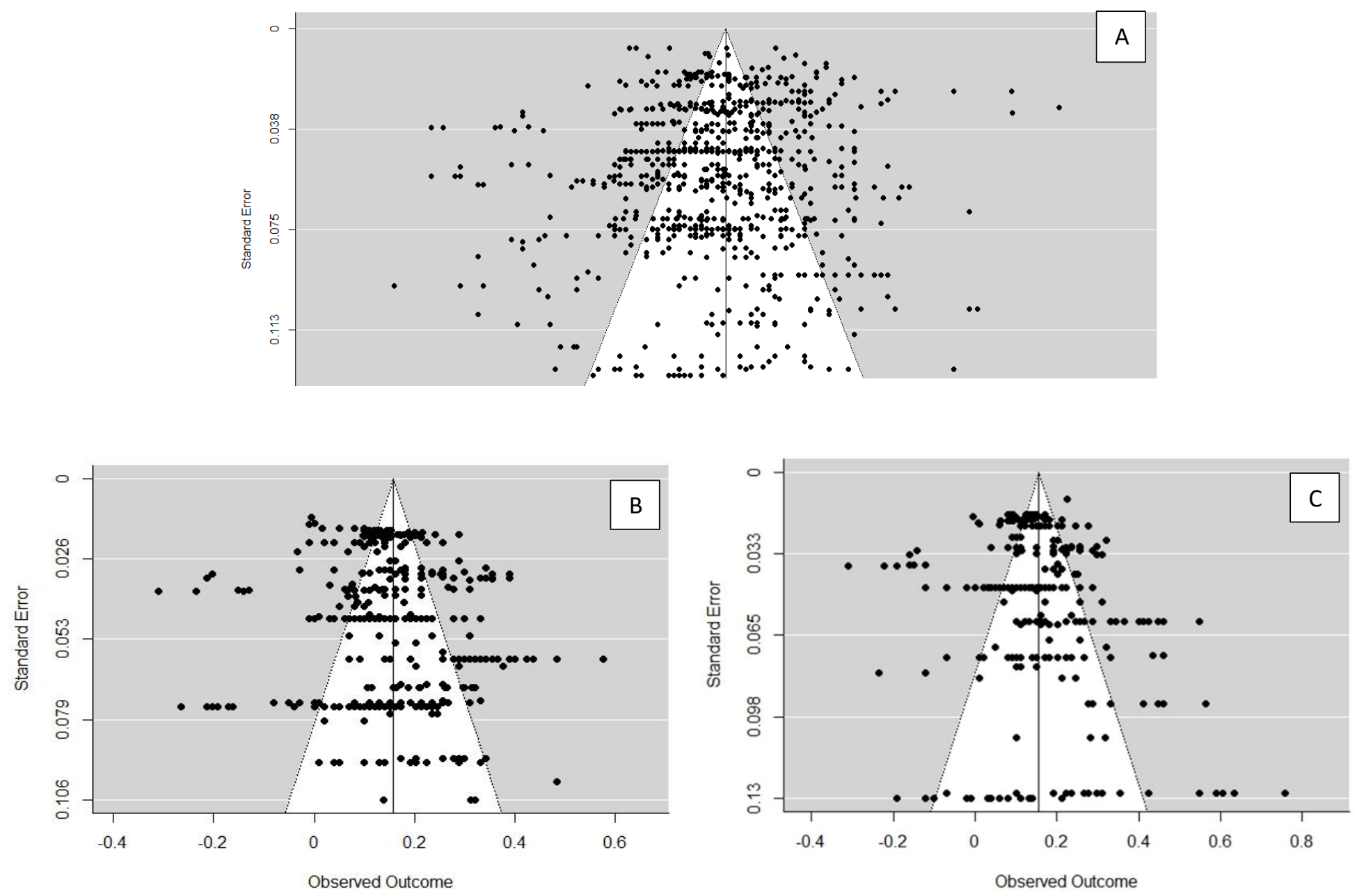

Figure 4. Funnel plots for the associations between parenting and self-control for cross-sectional (A), longitudinal parenting $\rightarrow$ self-control (B), and longitudinal self-control $\rightarrow$ parenting $(\mathrm{C})$. 
Table 1 Comparing cross-sectional and longitudinal effect sizes

\begin{tabular}{|c|c|c|c|c|c|}
\hline & $E S_{z}$ & S.E. & $t$ & $95 \% C I$ & $p$ \\
\hline$\Delta$ slope cross-sectional $\quad($ vs. $\mathrm{P} \rightarrow \mathrm{SC})$ & -.050 & .008 & -6.228 & $-.066,-.034$ & $<.001$ \\
\hline$\Delta$ slope cross-sectional $\quad($ vs. $\mathrm{SC} \rightarrow \mathrm{P})$ & -.052 & .009 & -6.058 & $-.068,-.035$ & $<.001$ \\
\hline$\Delta$ slope $\mathrm{P} \rightarrow \mathrm{SC}($ vs. SC $\rightarrow \mathrm{P})$ & -.002 & .009 & -0.174 & $-.019, .016$ & .862 \\
\hline$Q_{\mathrm{E}}(\mathrm{df})$ & \multicolumn{5}{|c|}{$18783.35(1537), p<.001$} \\
\hline Omnibus test & \multicolumn{5}{|c|}{$F(2,1537)=26.136, p<.001$} \\
\hline Variance level 2 & \multicolumn{5}{|c|}{$0.008, p<.001$} \\
\hline Variance level 3 & \multicolumn{5}{|c|}{$0.010, p<.001$} \\
\hline \# ES & \multicolumn{5}{|l|}{1540} \\
\hline
\end{tabular}


Table 2 Cross-sectional associations: the $Q_{E}$ statistics testing residual heterogeneity, and the Omnibus to test the effect of the moderators

\begin{tabular}{|c|c|c|c|c|}
\hline Moderator & $Q_{\mathrm{E}}(\mathrm{df})$ & $p$ & Omnibus test & $p$ \\
\hline \multicolumn{5}{|l|}{ Theoretical moderators } \\
\hline Age & $10420.318(830)$ & $<.001$ & $F(1,830)=1.632$ & .202 \\
\hline Ethnicity of adolescents & $9503.564(722)$ & $<.001$ & $F(5,722)=1.286$ & .268 \\
\hline Hofstede's individualism & $12883.359(862)$ & $<.001$ & $F(1,862)=0.300$ & .584 \\
\hline Type of parenting & $12763.277(873)$ & $<.001$ & $F(2,873)=3.483$ & .031 \\
\hline Parent gender & $13035.444(865)$ & $<.001$ & $F(2,865)=2.743$ & .065 \\
\hline Adolescent gender & $10777.295(840)$ & $<.001$ & $F(2,840)=1.443$ & .237 \\
\hline \multicolumn{5}{|l|}{ Methodological moderators } \\
\hline Informant of parenting measure & $13034.229(871)$ & $<.001$ & $F(3,871)=5.172$ & .002 \\
\hline Informant of self-control measure & $12859.771(872)$ & $<.001$ & $F(3,872)=5.068$ & .002 \\
\hline Consistency in informants & $13104.211(874)$ & $<.001$ & $F(1,874)=15.043$ & $<.001$ \\
\hline
\end{tabular}




Mype of parenting
positive parenting
negative parenting
parent-child relationship
$\Delta$ slope of positive (vs. negative)
$\Delta$ slope of positive (vs. parent-child relationship )
$\Delta$ slope of negative (vs. parent-child relationship )

$\begin{array}{ccccccc}\# E S & E S_{z} & S E & t & 95 \% C I & p & r \\ 876 & & & & & & \\ 446 & .213 & .011 & 18.826 & .191, .235 & <.001 & .210 \\ 290 & .188 & .013 & 14.864 & .163, .212 & <.001 & .186 \\ 140 & .220 & .016 & 13.676 & .188, .252 & <.001 & .217 \\ & -.025 & .010 & -2.444 & -.045,-.005 & .015 & \\ & .007 & .016 & 0.458 & -.024, .038 & .647 & \\ & .032 & .017 & 1.907 & -.001, .066 & .057 & \end{array}$

Report informant of parenting measure

adolescent self-report

other-report

observation

composite

$\Delta$ slope of adolescent self-report (vs. other-report)

$\Delta$ slope of adolescent self-report (vs. observation)

$\Delta$ slope of adolescent self-report (vs. composite)

$\Delta$ slope of other-report (vs. observation)

$\Delta$ slope of other-report (vs. composite)

$\Delta$ slope of observatoin (vs. composite)

$.007, .132$

$-.152,-.019$

.029

$.010, .143$

.011

.162

.034

2.259

$.081, .244$

.024

Report informant of self-control measure

adolescent self-report

other-report

observation

composite

$\Delta$ slope of adolescent self-report (vs. other-report) $\Delta$ slope of adolescent self-report (vs. observation) $\Delta$ slope of adolescent self-report (vs. composite)

$\begin{array}{cccc}546 & .203 & .011 & 18.667 \\ 251 & .188 & .017 & 11.161 \\ 23 & .121 & .039 & 3.092 \\ 56 & .297 & .029 & 10.291 \\ & -.015 & .017 & -0.918 \\ & -.082 & .039 & -2.08 \\ & .094 & .030 & 3.145 \\ & -.067 & .038 & -1.736 \\ & .109 & .033 & 3.313 \\ & .176 & .048 & 3.635\end{array}$

$$
\begin{aligned}
& .182, .224 \\
& .155, .221 \\
& .044, .198 \\
& .240, .353 \\
& -.048, .017 \\
& -.159,-.005 \\
& .035, .152 \\
& -.142, .009 \\
& .044, .173 \\
& .081, .270
\end{aligned}
$$

$\begin{array}{ll}<.001 & .200 \\ <.001 & .186 \\ .002 & .120 \\ <.001 & .289 \\ .359 & \\ .038 & \\ .002 & \\ .083 & \\ <.001 & \\ <.001 & \end{array}$




\section{Consistency of report informant}

consistent

inconsistent

$\Delta$ slope of consistent report informant (vs. inconsistent)
875

537

338

$\begin{array}{lll}.223 & .011 & 19.785 \\ .168 & .015 & 11.475 \\ -.056 & .014 & -3.903\end{array}$

$.201, .245$

$<.001$

219
139,196 $-.084,-.028$
$<.001$

$<.001$ 


\begin{tabular}{|c|c|c|c|c|}
\hline Moderator variables & $\beta(\mathrm{SE})$ & $95 \% \mathrm{CI}$ & $t$-Statistic & $p$-Value \\
\hline Intercept & $.221(.013)$ & $.196, .246$ & 17.389 & $<.001$ \\
\hline Type of parenting: negative parenting (vs. positive parenting) & $-.028(.010)$ & $-.048,-.007$ & -2.675 & .008 \\
\hline Type of parenting: parent-child relationship (vs. positive parenting) & $.005(.016)$ & $-.026, .036$ & 0.305 & .760 \\
\hline Informant of parenting: other-report (vs. self-report) & $-.008(.015)$ & $-.037, .021$ & -0.557 & .578 \\
\hline Informant of parenting: observation (vs. self-report) & $-.058(.034)$ & $-.125, .009$ & -1.713 & .087 \\
\hline Informant of parenting: composite (vs. self-report) & $.050(.034)$ & $-.017, .117$ & 1.465 & .143 \\
\hline Informant of self-control: other-report (vs. self-report) & $.013(.017)$ & $-.021, .047$ & 0.754 & .451 \\
\hline Informant of self-control: observation (vs. self-report) & $-.079(.041)$ & $-.158, .001$ & -1.936 & .053 \\
\hline Informant of self-control: composite (vs. self-report) & $.126(.033)$ & $.061, .191$ & 3.829 & $<.001$ \\
\hline Consistency of report informant: inconsistent (vs. consistent) & $-.069(.016)$ & $-.099,-.038$ & -4.422 & $<.001$ \\
\hline Omnibus test: & \multicolumn{4}{|c|}{$F(9,865)=6.157, p<.001$} \\
\hline Variance level 2 & \multicolumn{4}{|l|}{$.009, p<.001$} \\
\hline Variance level 3 & \multicolumn{4}{|l|}{$.011, p<.001$} \\
\hline \# ES & \multicolumn{4}{|l|}{875} \\
\hline
\end{tabular}


Table 5 Longitudinal association $P \rightarrow S C$ : the $Q_{E}$ statistics testing residual heterogeneity, and the Omnibus to test the effects of the moderators

\begin{tabular}{|c|c|c|c|c|}
\hline Moderator & $Q_{\mathrm{E}}(\mathrm{df})$ & $p$ & Omnibus test (3-digits) & $p$ \\
\hline \multicolumn{5}{|l|}{ Theoretical moderators } \\
\hline Ethnicity of adolescents & $2623.127(319)$ & $<.001$ & $F(5,319)=0.233$ & .948 \\
\hline Hofstede's individualism & $3345.279(371)$ & $<.001$ & $F(1,371)=0.422$ & .516 \\
\hline Type of Parenting & $3243.333(370)$ & $<.001$ & $F(2,370)=1.303$ & .273 \\
\hline Parent gender & $3188.953(366)$ & $<.001$ & $F(2,366)=6.150$ & .002 \\
\hline \multicolumn{5}{|l|}{ Methodological moderators } \\
\hline Informant parenting measure & $3132.167(360)$ & $<.001$ & $F(3,360)=3.770$ & .011 \\
\hline Consistency informants & $3186.39(371)$ & $<.001$ & $F(1,371)=6.562$ & .011 \\
\hline
\end{tabular}




\section{Moderators}$$
\text { \#ES }
$$

\#ES

Gender parent

Mother

Father

Both

$\Delta$ slope of mother (vs. father)

$\Delta$ slope of mother (vs. both)

$\Delta$ slope of father (vs. both)

Report informant of parenting measure

adolescent self-report

other-report

observation

composite

$\Delta$ slope of adolescent self-report (vs. other-report)

$\Delta$ slope of adolescent self-report (vs. observation)

$\Delta$ slope of adolescent self-report (vs. composite)

$\Delta$ slope of other-report (vs. observation)

$\Delta$ slope of other-report (vs. composite)

$\Delta$ slope of observatoin (vs. composite)

consistency of report informant

consistent

inconsistent

$\Delta$ slope of consistent report informant (vs. inconsistent)

Note. $*<.05, * *<.01, * * *<.001$

369

$129 \quad .19$

$47 \quad .181$

193

193

93

.129

$-.013$

$-.064$

$-.052$

364

171

17

62

44
87

$\begin{array}{cccccc}.152 & .018 & 8.554 & .117, .187 & <.001 & .151 \\ .150 & .021 & 7.013 & .108, .192 & <.001 & .149 \\ .110 & .030 & 3.54 & .050-.180 & <.001 & .110 \\ .218 & .025 & 8.881 & .169, .266 & <.001 & .215 \\ -.002 & .021 & -0.102 & -.044, .040 & .919 & .000 \\ -.040 & .034 & -1.188 & -.106, .026 & .236 & .040 \\ .065 & .023 & 2.874 & .021, .110 & .004 & .065 \\ -.038 & .033 & -1.159 & -.102, .026 & .247 & -.038 \\ .068 & .028 & 2.377 & .012, .124 & .018 & .068 \\ .105 & .037 & 2.853 & .033, .178 & .005 & -.105 \\ & & & & & \\ .138 & .017 & 8.259 & .105, .170 & <.001 & .137 \\ .179 & .017 & 10.610 & .146, .212 & <.001 & .177 \\ .041 & .016 & 2.562 & .010, .072 & .011 & .041\end{array}$


Intercept

Gender parent: father (vs. mother)

Gender parent: both (vs. mother)

Report informant of parenting measure: other report (vs. self-report)

Report informant of parenting measure: observation (vs. self-report)

Report informant of parenting measure: composite (vs. self-report)

Consistency of report informant: inconsistent (vs. consistent)

$\begin{array}{cccc}\beta(\mathrm{SE}) & 95 \% \mathrm{CI} & t \text {-Statistic } & p \text {-Value } \\ .179(.021) & .138, .221 & 8.514 & <.001 \\ -.016(.018) & -.051, .0191 & -0.900 & .369 \\ -.061(.020) & -.100,-.022 & -3.068 & .002 \\ -.022(.023) & -.067, .022 & -0.985 & .325 \\ -.068(.037) & -.140, .005 & -1.835 & .067 \\ .030(.026) & -.021, .081 & 1.461 & .253 \\ .043(.020) & .004, .081 & 2.188 & .029\end{array}$

Omnibus test

Variance level 2

Variance level 3
$F(6,353)=4.278, p<.001$

$.006, p<.001$

$.005, p<.001$

360 
Table 8 Longitudinal association $S C \rightarrow P:$ the QE statistics testing residual heterogeneity, and the Omnibus to test the effect of the moderators

\begin{tabular}{|c|c|c|c|c|}
\hline Moderator & $Q_{\mathrm{E}}(\mathrm{df})$ & $p$ & Omnibus test & $p$ \\
\hline \multicolumn{5}{|l|}{ Moderators of theoretical interest } \\
\hline Ethnicity of adolescents & $1881.894(246)$ & $<.001$ & $F(5,246)=1.303$ & .263 \\
\hline Hofstede's individualism & $2253.226(289)$ & $<.001$ & $F(1,289)=0.323$ & .570 \\
\hline Type of Parenting & $2263.720(288)$ & $<.001$ & $F(2,288)=0.122$ & .885 \\
\hline Gender of parents & $2264.139(287)$ & $<.001$ & $F(2,287)=2.354$ & .097 \\
\hline \multicolumn{5}{|l|}{ Methodological characteristics } \\
\hline Informant parenting measure & $1999.973(286)$ & $<.001$ & $F(3,286)=7.075$ & $<.001$ \\
\hline Consistency informants & $2231.285(289)$ & $<.001$ & $F(1,289)=1.100$ & .295 \\
\hline
\end{tabular}




\begin{tabular}{|c|c|c|c|c|c|c|c|}
\hline Moderators & \#ES & $E S_{z}$ & $S E$ & $t$ & $95 \% C I$ & $p$ & $r$ \\
\hline \multicolumn{8}{|l|}{ Informant Parenting } \\
\hline Self-report & 128 & .154 & .024 & 6.478 & $.107, .201$ & $<.001$ & .153 \\
\hline Other report & 34 & .096 & .026 & 3.626 & $.044, .148$ & $<.001$ & .096 \\
\hline Observation & 37 & .113 & .034 & 3.331 & $.046, .180$ & $<.001$ & .113 \\
\hline Composite & 91 & .232 & .035 & 6.684 & $.164, .300$ & $<.001$ & .228 \\
\hline$\Delta$ Slope self-report (vs. other report) & & -.058 & .018 & -3.303 & $-.093,-.024$ & .001 & -.058 \\
\hline$\Delta$ Slope self-report (vs. observation) & & -.041 & .036 & -1.130 & $-.112, .030$ & .260 & -.041 \\
\hline$\Delta$ Slope self-report (vs. composite) & & .078 & .039 & 1.994 & $.001, .154$ & .047 & .078 \\
\hline$\Delta$ Slope other-report (vs. observation) & & .018 & .037 & 0.478 & $-.055, .090$ & .633 & .018 \\
\hline$\Delta$ Slope other-report (vs. composite) & & .136 & .040 & 3.418 & $.058, .214$ & $<.001$ & .135 \\
\hline$\Delta$ Slope observation (vs. composite) & & .118 & .038 & 3.113 & $.044, .193$ & .002 & .117 \\
\hline Omnibus test & \multicolumn{7}{|c|}{$F(3,286)=7.075, p<.001$} \\
\hline Variance level 2 & \multicolumn{7}{|c|}{$.004, p<.001$} \\
\hline Variance level 3 & \multicolumn{7}{|c|}{$.010, p<.001$} \\
\hline \# ES & \multicolumn{7}{|l|}{290} \\
\hline
\end{tabular}


Table 10

Summary of overall effects and results of moderation analyses based on multiple moderator tests

\begin{tabular}{llll}
\hline & cross-sectional & longitudinal P $\rightarrow$ SC & Longitudinal SC $\rightarrow$ P \\
\hline Overall association $r$ & 0.204 & 0.157 & 0.155 \\
$\begin{array}{l}\text { Theoretical moderators } \\
\text { Ethnicity of adolescents }\end{array}$ & not significant & not significant & not significant \\
Hofstede's individualism & not significant & not significant & not significant \\
Type of parenting & positive $>$ negative parenting & not significant & not significant \\
Parent gender & not significant & both parents' assessed $<$ other & not significant \\
& not significant & not enough information & not enough information \\
Adolescent gender & not significant & not applicable & not applicable \\
Age & & & not significant \\
Methodological moderators & not significant & not enough information & composite effect size $>$ other three \\
Informant of parenting & composite $>$ other three & informants \\
measure & informants & not enough information \\
Informant of self-control & consistent $>$ inconsistent & inconsistent $>$ consistent & not significant \\
measure & & &
\end{tabular}


Table 11 Recommendation of sample size

\begin{tabular}{|c|c|c|c|c|}
\hline & $\begin{array}{c}\alpha=.05 ; \text { power }=.80 ; \\
\text { two-tailed }\end{array}$ & $\begin{array}{c}\alpha=.05 ; \text { power }=.90 ; \\
\text { two-tailed }\end{array}$ & $\begin{array}{c}\alpha=.05 ; \text { power }=.95 ; \\
\text { two-tailed }\end{array}$ & $\begin{array}{c}\alpha=.05 ; \text { power }=.99 ; \\
\text { two-tailed }\end{array}$ \\
\hline 1. Cross-sectional design $\left(E S_{r}=.204\right)$ & 186 & 248 & 306 & 432 \\
\hline 2. Longitudinal design: parenting $\rightarrow$ self-control $\left(E S_{r}=.157\right)$ & 316 & 422 & 521 & 736 \\
\hline 3. Longitudinal design: self-control $\rightarrow$ parenting $\left(E S_{r}=.155\right)$ & 324 & 433 & 535 & 755 \\
\hline
\end{tabular}

Note. Software version: G*Power 3.1.9.2; Test family: exact; Statistical test: correlation: bivariate normal model. 
Supplemental Table 1: Details on parenting categories, with numbers representing the number of effect sizes within the category

\begin{tabular}{|c|c|c|c|c|}
\hline Parenting Term & Positive & Negative & Relationship & Total \\
\hline Monitoring & 137 & & & \\
\hline Support & 133 & & & \\
\hline Authoritative & 139 & & & \\
\hline Nurturing, responsive discipline & 95 & & & \\
\hline Warmth & 81 & & & \\
\hline Autonomy & 17 & & & \\
\hline Supervision & 53 & & & \\
\hline Respectful positive control & 46 & & & \\
\hline Acceptance & 21 & & & \\
\hline Sensitivity & 24 & & & \\
\hline Positive expressivity & 25 & & & \\
\hline Cohesion & 6 & & & \\
\hline Involvement & 33 & & & \\
\hline Conflict & & 117 & & \\
\hline Psychological negative control & & 81 & & \\
\hline Authoritarian & & 97 & & \\
\hline Harsh & & 79 & & \\
\hline Hostility & & 16 & & \\
\hline Permissive & & 43 & & \\
\hline Coercion & & 7 & & \\
\hline Rejection & & 23 & & \\
\hline Withdrawal & & 14 & & \\
\hline Abuse & & 26 & & \\
\hline Negativity & & 10 & & \\
\hline Attachment & & & 108 & \\
\hline Relationship & & & 47 & \\
\hline Closeness & & & 16 & \\
\hline Bond & & & 21 & \\
\hline Communication & & & 12 & \\
\hline Trust & & & 8 & \\
\hline Alienation & & & 5 & \\
\hline Total number of effect sizes & & & & 1540 \\
\hline
\end{tabular}

